\title{
Spectroscopic and photometric studies of low-metallicity star-forming dwarf galaxies
}

\author{
III. SBS $1415+437$
}

\author{
N. G. Guseva ${ }^{1}$, P. Papaderos ${ }^{2}$, Y. I. Izotov ${ }^{1}$, R. F. Green ${ }^{3}$, K. J. Fricke ${ }^{2}$, T. X. Thuan ${ }^{4}$, and K. G. Noeske ${ }^{2}$ \\ 1 Main Astronomical Observatory, Ukrainian National Academy of Sciences, Zabolotnoho 27, Kyiv 03680, Ukraine \\ 2 Universitäts-Sternwarte, Geismarlandstraße 11, 37083 Göttingen, Germany \\ 3 National Optical Astronomy Observatory, Tucson, AZ 85726, USA \\ 4 Astronomy Department, University of Virginia, Charlottesville, VA 22903, USA
}

Received 21 November 2002 / Accepted 16 May 2003

\begin{abstract}
We present a detailed optical spectroscopic and $B, V, I, \mathrm{H} \alpha$ photometric study of the metal-deficient cometary blue compact dwarf $(\mathrm{BCD})$ galaxy SBS $1415+437$. We derive an oxygen abundance $12+\log (\mathrm{O} / \mathrm{H})=7.61 \pm 0.01$ and $7.62 \pm 0.03$ $\left(Z=Z_{\odot} / 20\right)^{\star}$ in the two brightest $\mathrm{H}$ II regions, among the lowest in BCDs. The helium mass fractions in these regions are $Y=0.246 \pm 0.003$ and $0.243 \pm 0.010$. Four techniques based on the equivalent widths of the hydrogen emission and absorption lines, the spectral energy distribution and the colours of the galaxy are used to put constraints on the age of the stellar population in the low-surface-brightness (LSB) component of the galaxy, assuming two limiting cases of star formation (SF), the case of an instantaneous burst and that of a continuous SF with a constant or a variable star formation rate (SFR). The spectroscopic and photometric data for different regions of the LSB component are well reproduced by a young stellar population with an age $t \leq 250 \mathrm{Myr}$, assuming a small extinction in the range $A_{V}=0-0.6 \mathrm{mag}$. Assuming no extinction, we find that the upper limit for the mass of the old stellar population, formed between $2.5 \mathrm{Gyr}$ and $10 \mathrm{Gyr}$, is not greater than $\sim(1 / 20-1)$ of that of the stellar population formed during the last $\sim 250 \mathrm{Myr}$. Depending on the region considered, this also implies that the SFR in the most recent SF period must be 20 to 1000 times greater than the SFR at ages $\gtrsim 2.5$ Gyr. We compare the photometric and spectroscopic properties of SBS $1415+437$ with those of a sample of 26 low-metallicity dwarf irregular and BCD galaxies. We show that there is a clear trend for the stellar LSB component of lower-metallicity galaxies to be bluer. This trend cannot be explained only by metallicity effects. There must be also a change in the age of the stellar populations. The most metal-deficient galaxies have also smaller luminosity-weighted ages.
\end{abstract}

Key words. galaxies: abundances - galaxies: dwarf - galaxies: evolution - galaxies: compact - galaxies: starburst galaxies: stellar content - galaxies: individual: SBS 1415+437

\section{Introduction}

Since its discovery as a metal-deficient blue compact dwarf (BCD) galaxy (Thuan et al. 1995), SBS 1415+437 (ECG 389) has been considered as a probable nearby young dwarf galaxy. Situated at a distance $D=11.4 \mathrm{Mpc}$ it was classified by Thuan et al. (1999) as a cometary BCD with a very bright supergiant $\mathrm{H}$ II region at the SW tip of the galaxy.

From $4 \mathrm{~m}$ Kitt Peak National Observatory (KPNO) telescope spectra, Thuan et al. (1995) first derived an oxygen abundance of $12+\log (\mathrm{O} / \mathrm{H})=7.51 \pm 0.01$ in SBS $1415+437$ placing the galaxy among the most metal-deficient BCDs known. Later, Izotov \& Thuan $(1998,1999)$ derived from the same spectrum $12+\log (\mathrm{O} / \mathrm{H})=7.59 \pm 0.01$ using five-level atom

Send offprint requests to: N. G. Guseva,

e-mail: guseva@mao.kiev.ua

$\star 12+\log (\mathrm{O} / \mathrm{H})_{\odot}=8.92$ (Anders \& Grevesse 1989). models for abundance determination instead of the three-level atom model used by Thuan et al. (1995). Thuan et al. (1999), using Multiple Mirror Telescope (MMT) and Hubble Space Telescope $(H S T)$ FOS observations, derived $12+\log (\mathrm{O} / \mathrm{H})=$ $7.60 \pm 0.01$ and $7.54 \pm 0.14$, respectively. The high brightness and low metallicity of the H II region in SBS $1415+437$ make this galaxy one of the best objects for helium abundance determination. Izotov \& Thuan (1998) and Thuan et al. (1999) derived respectively a helium mass fraction $Y=0.244 \pm 0.002$ and $0.246 \pm 0.004$ for it, close to the primordial helium mass fraction of $Y_{\mathrm{p}}=0.245 \pm 0.002$ by Izotov et al. (1999).

Thuan et al. (1999) have discussed the evolutionary status of SBS 1415+437, using ground-based MMT spectroscopic and $H S T$ /WFPC2 photometric data. Based on the $(V-I)$ vs. $I$ colour-magnitude diagrams (CMD) and spectral energy distributions (SED) in the optical range, they concluded that SBS $1415+437$ is a truly young galaxy that did not start to 
form stars until $\sim 100$ Myr ago. However, the $V$ and $I$ images used by Thuan et al. (1999) were not deep enough for the detection of old red giant branch (RGB) stars in the CMD. Furthermore, they considered an instantaneous burst model which gives only a lower limit to the age of the stellar population in SBS $1415+437$.

In this paper we combine new spectroscopic and photometric data with previous observations to derive elemental abundances and to better constrain the age of the stellar population in SBS 1415+437. For the latter task we use four different techniques of age determination and consider different star formation (SF) histories. The paper is organized as follows. In Sect. 2 we describe the observations and data reduction. The photometric properties of SBS $1415+437$ are described in Sect. 3 . We derive in Sect. 4 the chemical abundances in the two brightest $\mathrm{H}$ II regions. In Sect. 5 we discuss the properties of the stellar populations in SBS $1415+437$ and compare them with those in other low-metallicity dwarf galaxies. Finally, Sect. 6 summarises the main conclusions of this study.

\section{Observations and data reduction}

\subsection{Photometry}

Narrow-band images of SBS $1415+437$ in the $\mathrm{H} \alpha$ line at $\lambda 6563 \AA$ through a passband with a full width at half maximum $(F W H M)$ of $74 \AA$, and in the adjacent continuum at $\lambda 6477 \AA$ through a passband with $F W H M=72 \AA$ were obtained with the Kitt Peak ${ }^{1} 2.1 \mathrm{~m}$ telescope on April 22, 1999 during a photometric night. The telescope was equipped with a Tektronix $1024 \times 1024 \mathrm{CCD}$ detector operating at a gain of $3 \mathrm{e}^{-} \mathrm{ADU}^{-1}$, giving an instrumental scale of 0. .' $^{\prime} 305$ pixel $^{-1}$ and field of view of $5^{\prime}$. The total exposures of $50 \mathrm{~min}$ in the $\mathrm{H} \alpha$ line and $40 \mathrm{~min}$ in the adjacent continuum bluewards of $\mathrm{H} \alpha$ were split up into 5 and 4 subexposures, slightly offset with respect to each other for removal of cosmic particle hits and bad pixels. The point spread function has a FWHM of 2!'2. Bias and flatfield frames were obtained during the same night. The standard stars Feige 34 and HZ 44 were observed in both filters during the same night at several airmasses for absolute flux calibration.

Another broad-band $B$ image (15 min) of SBS 1415+437 was obtained on March 9, 1997 under photometric conditions, with the CAFOS focal reducer attached to the $2.2 \mathrm{~m}$ telescope of the German-Spanish Astronomical Center, Calar Alto ${ }^{2}$, Spain. CAFOS was equipped with a SITe $2048 \times 2048$ CCD operating at a gain of $2.3 \mathrm{e}^{-} \mathrm{ADU}^{-1}$, with a read-out noise of $<3$ counts (rms). With a focal ratio of $f / 4.4$, the instrumental scale was 0 . $^{\prime} 53$ pixel $^{-1}$ and field of view $\sim 15^{\prime}$. The seeing during the observations was 3.'1 (FWHM). Standard stars from Christian et al. (1985) were observed at several airmasses for calibration.

\footnotetext{
${ }^{1}$ Kitt Peak National Observatory (KPNO) is operated by the Association of Universities for Research in Astronomy (AURA), Inc., under cooperative agreement with the National Science Foundation (NSF).

${ }^{2}$ German-Spanish Astronomical Center, Calar Alto, operated by the Max-Planck-Institute for Astronomy, Heidelberg, jointly with the Spanish National Commission for Astronomy.
}

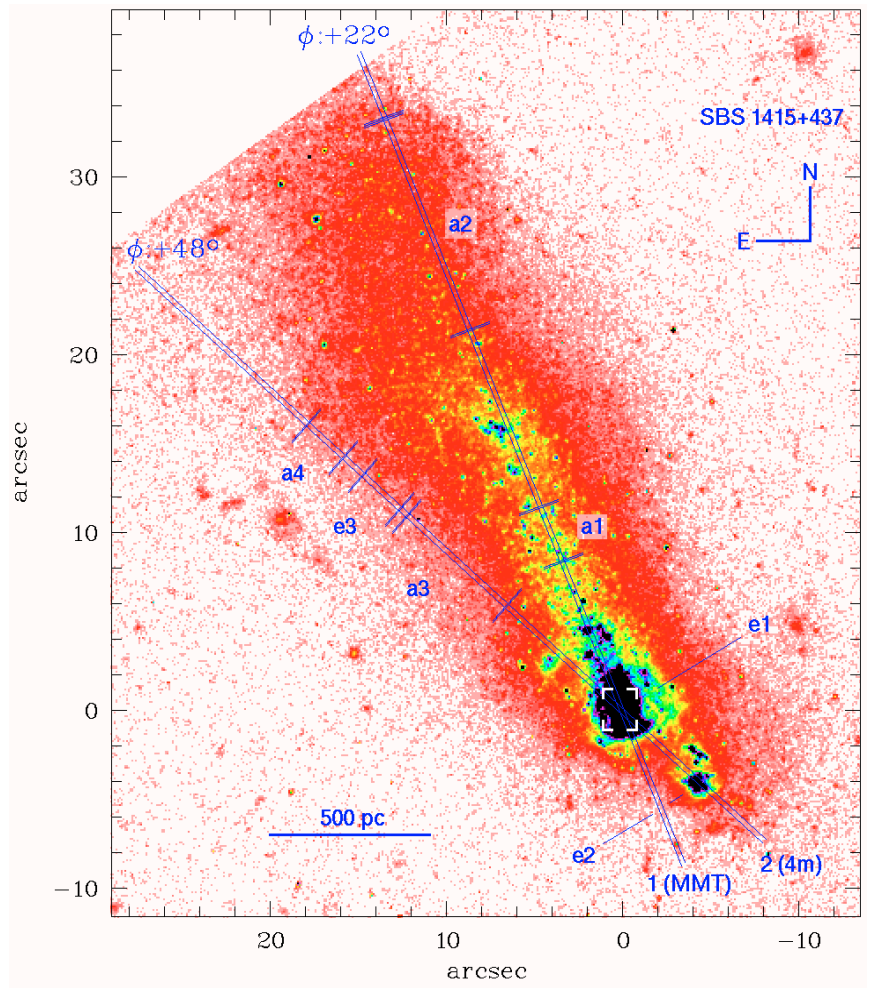

Fig. 1. HST $V$ image of SBS $1415+437$. The long-slit positions during the two observations with the MMT and $4 \mathrm{~m}$ Mayall telescope are labeled "1" and "2", respectively. North is up and east is to the left. Regions e1-e3 with hydrogen emission lines in the spectra and regions a1-a4 with hydrogen absorption lines are labeled.

Standard reduction steps, including bias subtraction, flatfield correction, removal of cosmic particle hits and absolute flux calibration were carried out using IRAF $^{3}$ and MIDAS ${ }^{4}$.

The ground-based photometric data were supplemented by the HST/WFPC2 $V(\mathrm{~F} 569 \mathrm{~W})$ and $I(\mathrm{~F} 791 \mathrm{~W})$ images described by Thuan et al. (1999).

\subsection{Spectroscopy}

Spectroscopic observations were carried out on June 18, 1999, at the Kitt Peak $4 \mathrm{~m}$ Mayall telescope with the Ritchey-Chrétien spectrograph and a T2KB $2048 \times 2048$ CCD detector. The $2^{\prime \prime} \times 300^{\prime \prime}$ slit was centered on the brightest $\mathrm{H}$ II region e1 (slit 2 in Fig. 1) with position angle $\mathrm{PA}=48^{\circ}$ so as to include the second brightest $\mathrm{H}$ II region e2 to the SW of region e1. We used the KPC-10A grating in first order and a GG 375 order separation filter. The spatial scale along the slit

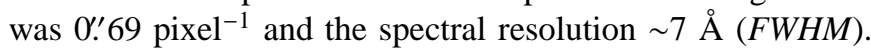
The spectra were obtained at an airmass 1.27 . The total exposure time of $60 \mathrm{~min}$ was broken up into 3 subexposures. No correction for atmospheric refraction was made because of the small airmass during the observations. Two Kitt Peak

\footnotetext{
${ }^{3}$ IRAF is the Image Reduction and Analysis Facility distributed by the National Optical Astronomy Observatory, which is operated by the AURA under cooperative agreement with the NSF.

${ }^{4}$ Munich Image Data Analysis System, provided by the European Southern Observatory (ESO).
} 


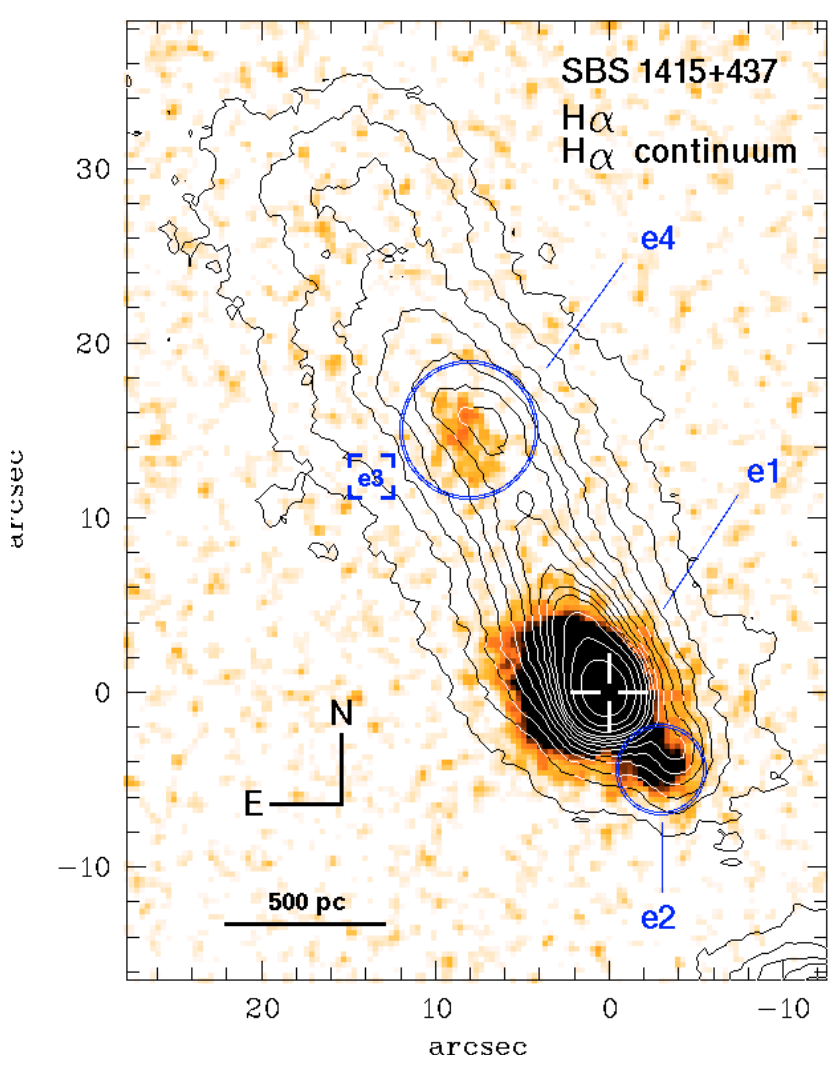

Fig. 2. Continuum-subtracted H $\alpha$ image of SBS $1415+437$ with superposed $\mathrm{H} \alpha$ continuum isophotes. Regions with nebular emission are labeled e1, e 2 and e 4 . The faint region e 3 with $\mathrm{H} \beta$ and $\mathrm{H} \alpha$ emission lines in its spectrum is not seen in the $\mathrm{H} \alpha$ image.

spectrophotometric standard stars were observed for flux calibration. For wavelength calibration, He-Ne-Ar comparison spectra were obtained after each exposure.

The data reduction was performed with the IRAF software package. This includes bias-subtraction, flat-field correction, cosmic-ray removal, wavelength calibration, night sky background subtraction, correction for atmospheric extinction and absolute flux calibration of the two-dimensional spectrum.

For abundance determination, one-dimensional spectra of regions e 1 and e 2 were extracted within apertures of $2^{\prime \prime} \times 4$.' 6 and $2^{\prime \prime} \times 4$.' 0 , respectively. In addition, we extracted spectra of the low-surface-brightness (LSB) regions a3 and a4 with strong hydrogen Balmer absorption lines, and of region e 3 with $\mathrm{H} \alpha$ and $\mathrm{H} \beta$ in emission (Fig. 1).

We also used the two-dimensional MMT spectrum obtained by Thuan et al. (1999) with the slit oriented at PA $=22^{\circ}$ (slit 1 in Fig. 1). We extracted one-dimensional spectra of the LSB regions a1 and a2 within apertures of $1 .{ }^{\prime \prime} 5 \times 3$ ". 4 and 1". $5 \times 13^{\prime \prime}$. 2 , respectively (Fig. 1). These spectra show strong hydrogen Balmer absorption lines.

The selected LSB regions are listed in Tables 3 and 4 with their positions and spatial extents. Origins are set at the center of the brightest region e1 (Figs. 1,2). The spectra of the LSB regions are used to study the stellar populations and to constrain the age of the oldest stars which contribute to the light of these regions.

\section{Photometric properties}

It is seen from the continuum-subtracted $\mathrm{H} \alpha$ images (Fig. 2) that star-forming activity in SBS $1415+437$ is primarily occurring in regions e 1 and $\mathrm{e} 2$, with some additional faint $\mathrm{H} \alpha$ emission present in region e4. However, the available narrow-band data are not deep enough for tracing faint $\mathrm{H} \alpha$ emission in other regions of the LSB component, such as in regions a1, a3 and 3 where $\mathrm{H} \alpha$ and $\mathrm{H} \beta$ have been detected spectroscopically (Table 3). The latter fact suggests that some low-level SF may be present at various locations within the LSB component.

The photometric properties of the stellar LSB component of SBS $1415+437$ were first investigated by Thuan et al. (1999) using HST/WFPC2 $V$ and $I$ images. These authors fit the surface brightness profiles (SBPs) of SBS $1415+437$ with an exponential distribution in the radius range $4^{\prime \prime} \leq R^{*} \lesssim 13^{\prime \prime}$ with a scale length $\alpha=5 . \prime 4(\approx 0.3 \mathrm{kpc})$. However, their study was limited by the fact that the HST images do not include the outermost NE part of the LSB component. Furthermore, the SBPs in Thuan et al. (1999) reach only a surface brightness level $\mu \sim 24.5 \mathrm{mag} \operatorname{arcsec}^{-2}$. It is known, however, that the star-forming component may contribute to the optical BCD emission to fainter surface brightness levels (see e.g. Papaderos et al. 2002 and references therein). A comparatively young stellar population has been observed in the inner part of the LSB component of other cometary BCDs, several hundred pc away from the brightest H II region (Noeske et al. 2000; Guseva et al. 2001; Fricke et al. 2001). Therefore deeper images are needed to study the outer parts of the LSB component in SBS $1415+437$.

Note that the SBPs by Thuan et al. (1999) show in the outermost part $\left(13^{\prime \prime} \lesssim R^{*} \lesssim 16^{\prime \prime}\right.$, or $\left.24 \lesssim \mu_{V} \lesssim 24.5\right)$ a steeper exponential intensity decrease than the one observed at intermediate intensity levels. This slope change, not discussed in Thuan et al. (1999), is found independently by us in the HST/WFPC2 SBPs derived with the method iv of Papaderos et al. (2002) and ellipse fitting to the visible part of the LSB component. While the latter method extends surface photometry out to larger radii $\left(R^{*} \sim 22^{\prime \prime}\right)$, it is subject to large uncertainties because the NE part of the LSB component with $\mu \gtrsim$ $24.5 V$ mag $\operatorname{arcsec}^{-2}\left(R^{*} \gtrsim 16^{\prime \prime}\right)$ lies outside the HST/WFPC2 field of view. Additionally, the outermost LSB isophotes show considerable deviations from ellipticity.

To study the surface brightness distribution at large radii we use the ground-based Calar Alto $B$ image. Despite the poor spatial resolution this image allows us to study the entire LSB component out to its Holmberg radius. The change in the exponential slope for $13^{\prime \prime} \lesssim R^{*} \lesssim 16^{\prime \prime}$ is confirmed from the ground-based $B$ SBP. At large radii, however, this SBP reveals a flatter, outer exponential regime with a scale length $\alpha$ fairly comparable to that previously obtained at intermediate intensity levels from HST data (upper thick-grey line in Fig. 3a). From fitting an exponential model to the $B$ SBP for $R^{*} \geq 16^{\prime \prime}$ (lower thick-grey line in Fig. 3a) we obtain a central surface brightness $\mu_{\mathrm{E}, 0}=21.37 \mathrm{~B}$ mag $\operatorname{arcsec}^{-2}$ and a scale length $\alpha=0.27 \mathrm{kpc}$.

Note, however, that the inner exponential profile studied by Thuan et al. (1999) is $\gtrsim 0.3$ mag brighter than the outer one, 


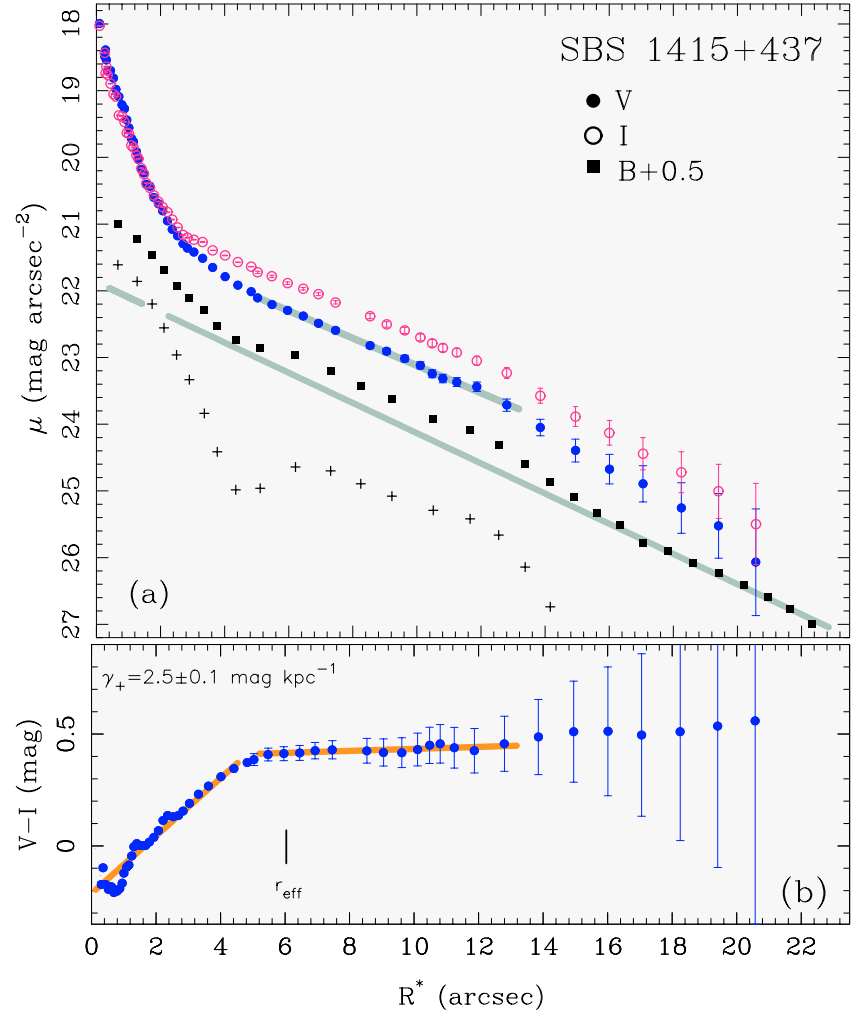

Fig. 3. a) Surface brightness profiles (SBPs) of SBS $1415+437$ in $V$ and $I$ (filled and open circles, respectively), derived from $H S T$ /WFPC2 data. A linear fit to the $V$ SBP in the radius interval $4^{\prime \prime} \leq R^{*} \lesssim 13^{\prime \prime}$ is shown by the upper solid-grey line. Both SBPs reveal a significant slope change for $R^{*} \gtrsim 13^{\prime \prime}$. Filled squares show the ground-based $B$ SBP, shifted vertically by $0.5 \mathrm{mag}$. In addition to the exponential intensity slope at radii $4^{\prime \prime} \leq R^{*} \lesssim 13^{\prime \prime}$, this profile reveals for $R^{*} \gtrsim 16^{\prime \prime}$ an outer exponential regime with an $\alpha \sim 0.27 \mathrm{kpc}$ (lower solid-grey line). The residuals after subtraction of the outer exponential distribution from the $B$ SBP are shown by crosses. b) $(V-I)$ colour profile of SBS 1415+437, computed from subtraction of the SBPs in a). A strong colour gradient $\gamma_{+}=2.5 \mathrm{mag} \mathrm{kpc}^{-1}$ is derived for radii smaller than the effective radius $r_{\text {eff }}$ (inner fit). The $(V-I)$ colour of $0.4-$ $0.5 \mathrm{mag}$ for larger radii is nearly constant with a $\gamma_{+}<0.2 \mathrm{mag} \mathrm{kpc}^{-1}$. Comparison of the $B$ and $V$ SBPs implies a $B-V$ color $\sim 0.2$ mag in the radius range $13^{\prime \prime} \leq R^{*} \leq 16^{\prime \prime}$.

which suggests that more than $1 / 4$ of the emission associated with this profile originates from the part of the LSB component between regions e1 and a2.

The present data provide no compelling evidence for a large age difference between the stellar population which dominates within the inner exponential regime discussed in Thuan et al. (1999) and that responsible for the outermost LSB emission (i.e. for $\left.R^{*}>16^{\prime \prime}\right)$. The $(V-I)$ profile reveals a strong colour gradient $\left(\gamma_{+}=2.5 \mathrm{mag} \mathrm{kpc}^{-1}\right.$; inner solid-grey line in Fig. 3b) within the inner $5^{\prime \prime}$, or roughly the $V$ band effective radius $\left(r_{\text {eff }}=5^{\prime \prime} 6\right)$ of SBS $1415+437$. At larger radii, however, linear fits to the $(V-I)$ profile yield, depending on whether they are error-weighted or not, a gradient not exceeding 0.1 and $0.2 \mathrm{mag} \mathrm{kpc}^{-1}$, respectively. Comparable values are also found from subtraction of the exponential fits in $V$ and $I$ (Fig. 3a) in the radius range $r_{\text {eff }} \leq R^{*} \leq 13^{\prime \prime}$ (Fig. 3b, solid-grey line at intermediate radii).

\section{Chemical abundances}

In this section we derive the elemental abundances of regions e 1 and e 2 using the Kitt Peak 4 m telescope observations. Their spectra with strong emission lines are shown in Fig. 4.

The observed $(F(\lambda))$ and extinction-corrected $(I(\lambda))$ emission line fluxes relative to the $\mathrm{H} \beta$ emission line fluxes, their equivalent widths $E W$, the extinction coefficients $C(\mathrm{H} \beta)$, the observed fluxes of the $\mathrm{H} \beta$ emission line, and the equivalent widths of the hydrogen absorption lines for regions e1 and e2 are shown in Table 1. Despite the differences in aperture $\left(2^{\prime \prime} \times 44^{\prime \prime} 6\right.$ for the Kitt Peak $4 \mathrm{~m}$ data, $1^{\prime \prime} 5 \times 5^{\prime \prime}$ and $1^{\prime \prime} .5 \times 0$. . $^{\prime} 6$ for the MMT data from Thuan et al. 1999), the relative fluxes of the emission lines for region $\mathrm{e} 1$ are in agreement within the errors with those derived by Thuan et al. (1999).

The physical conditions and heavy element abundances in regions e1 and e2 were derived following Izotov et al. (1994, 1997a) and Thuan et al. (1995). The electron temperatures $T_{\mathrm{e}}(\mathrm{O}$ III $), T_{\mathrm{e}}(\mathrm{S}$ III $), T_{\mathrm{e}}(\mathrm{O}$ II) for the high-, intermediateand low-ionization regions, respectively, the electron number densities $N_{\mathrm{e}}(\mathrm{S}$ II), ionization correction factors (ICF), and ionic and total heavy element abundances are shown in Table 2 for both regions. The oxygen abundance $12+\log (\mathrm{O} / \mathrm{H})=7.61 \pm$ $0.01\left(Z_{\odot} / 20\right)$ and heavy element abundance ratios for region e1 are in good agreement with those derived by Thuan et al. (1999). The oxygen abundance $12+\log (\mathrm{O} / \mathrm{H})=7.62 \pm 0.03$ and heavy element abundance ratios in region e 2 are consistent with those for region e1 within the errors.

In Fig. 5 we compare the heavy element abundance ratios in the two brightest regions of SBS $1415+437$ with data for a sample of low-metallicity BCDs. The $\mathrm{Ne} / \mathrm{O}, \mathrm{S} / \mathrm{O}, \mathrm{Ar} / \mathrm{O}$ and $[\mathrm{O} / \mathrm{Fe}]$ abundance ratios for the comparison sample are taken from Izotov \& Thuan (1999), while the $\mathrm{Cl} / \mathrm{O}$ abundance ratios are collected from Izotov \& Thuan (1998) and Izotov et al. (1997a). The heavy element abundance ratios for regions e1 (large squares) and e2 (small squares) are in good agreement with those for other BCDs. Note, that the $\mathrm{Cl} / \mathrm{O}$ ratio does not show any significant increase with increasing oxygen abundance. This conclusion is strengthened by the observations of Esteban et al. (1998, 1999a, 1999b) who derived $\log (\mathrm{Cl} / \mathrm{O})$ in the range from -3.28 to -3.47 for high-metallicity $\mathrm{H}$ II regions in Orion, M17 and M8 with $12+\log (\mathrm{O} / \mathrm{H})=8.60,8.50$ and 8.60 , respectively. For comparison, $\log (\mathrm{Cl} / \mathrm{O})=-3.37$ is derived for the Sun (Anders \& Grevesse 1989).

The high brightness of regions e1 and e 2 allows for a reliable determination of the ${ }^{4} \mathrm{He}$ abundance. Nine He I emission lines are detected in the spectrum of region e1 (Table 1). Two of them, He I $\lambda 3889$ and $\lambda 4713$, are blended with other lines. Six He I lines are detected in region e2. The five brightest He I $\lambda 3889, \lambda 4471, \lambda 5876, \lambda 6678, \lambda 7065$ emission lines are used to correct their fluxes for collisional and fluorescent enhancement. This is done by minimizing the deviations of the corrected He I line flux ratios from the recombination ratios, through varying the electron number density in the $\mathrm{He}^{+}$zone and the optical depth in the He I $\lambda 3889$ emission line. The flux of 


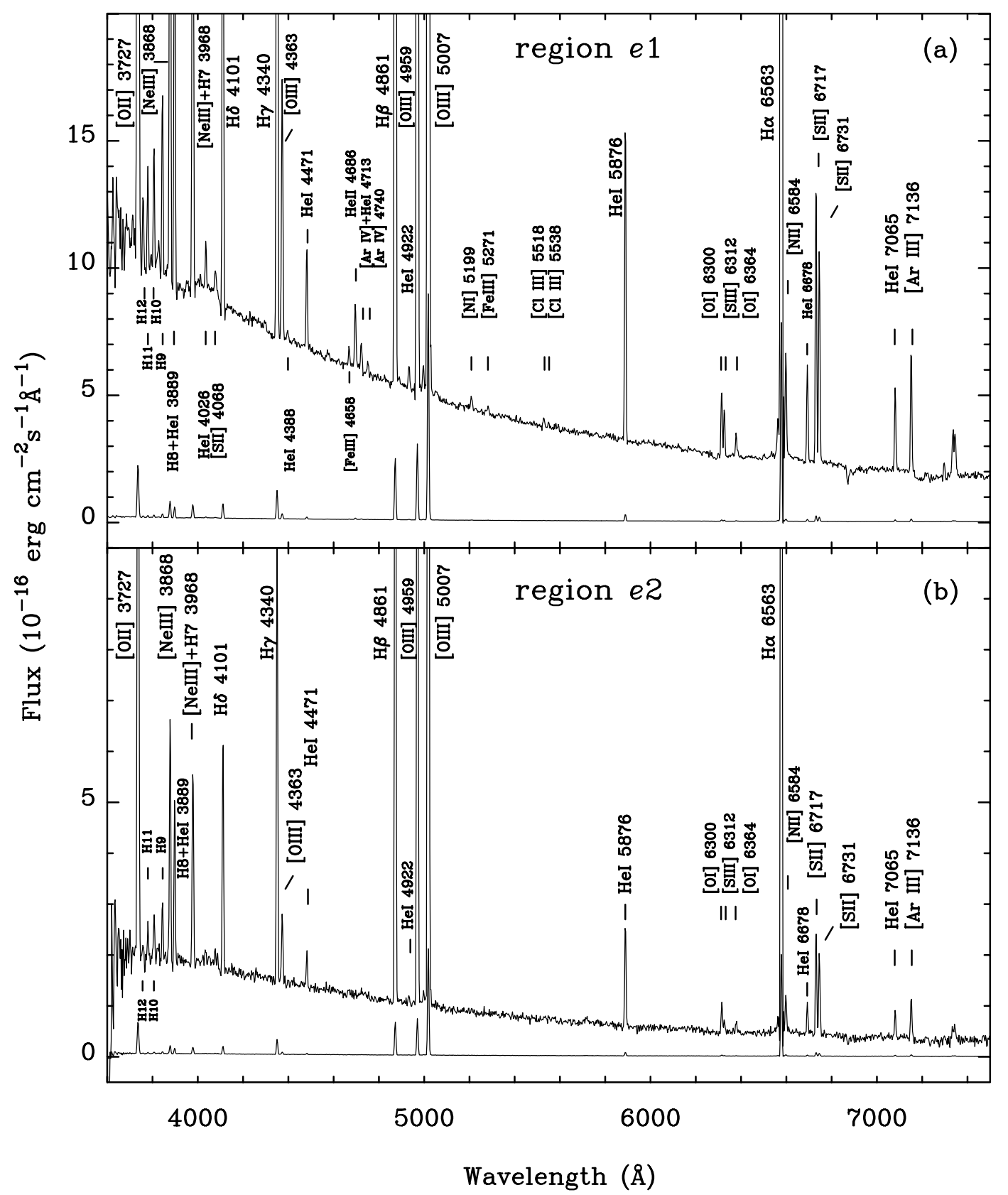

Fig. 4. The KPNO $4 \mathrm{~m}$ telescope spectra of the brightest $\mathrm{H}$ II regions e1 and e 2 with the identified emission lines. The lower spectra in a) and b) are the observed spectra downscaled by factors of 50 and 30, respectively.

this line was preliminarily corrected for the contribution of the H I $\lambda 3889$ emission line, according to prescriptions of Izotov et al. (1994, 1997a). Helium abundances $\mathrm{He}^{+} / \mathrm{H}^{+}$, derived from the corrected He I $\lambda 4471, \lambda 5876, \lambda 6678$ line fluxes and their weighted mean are listed in Table 2 . The abundance $\mathrm{He}^{+2} / \mathrm{H}^{+}$ is added to $\mathrm{He}^{+} / \mathrm{H}^{+}$for region e1, as He II $\lambda 4686$ is present in its spectrum. Note the lower He abundance derived from the He I $\lambda 4471$ flux which is most likely due to significant underlying stellar He I $\lambda 4471$ absorption. The effect of underlying absorption for the other He I emission lines used in the He abundance determination is much smaller, as they have much larger equivalent widths compared to the $\mathrm{He}$ I $\lambda 4471$ emission line (Table 1). The mean ${ }^{4} \mathrm{He}$ mass fractions $Y=0.246 \pm 0.003$ and $0.243 \pm 0.010$ in regions e 1 and e2 (Table 2) are consistent with the values derived for SBS $1415+437$ by Izotov \& Thuan (1998) and Thuan et al. (1999). They are also consistent with the primordial ${ }^{4} \mathrm{He}$ mass fraction $Y_{\mathrm{p}}=0.244 \pm 0.002$, derived by extrapolating the $Y$ vs. $\mathrm{O} / \mathrm{H}$ linear regression to $\mathrm{O} / \mathrm{H}=0$ (Izotov \& Thuan 1998), or to $Y_{\mathrm{p}}=0.245 \pm 0.002$ derived from spectroscopic observations of the two most metal-deficient BCDs known, I Zw 18 and SBS 0335-052 (Izotov et al. 1999).

\section{Age of the stellar population in the LSB regions}

We consider next the spectroscopic and photometric properties of the LSB regions labeled a1, a2 (slit position 1 in Fig. 1) and a3, e3, a4 (slit position 2), to constrain the age of the stellar populations contributing to the light in those regions. 
Table 1. Observed $(F(\lambda))$ and extinction-corrected $(I(\lambda))$ fluxes and equivalent widths $(E W)$ of emission lines in the $\mathrm{H}$ II regions e1 and e2.

\begin{tabular}{|c|c|c|c|c|c|c|}
\hline \multirow[b]{2}{*}{$\lambda_{0}(\AA)$ Ion } & \multicolumn{3}{|c|}{ region e1 } & \multicolumn{3}{|c|}{ region e2 } \\
\hline & $F(\lambda) / F(\mathrm{H} \beta)$ & $I(\lambda) / I(\mathrm{H} \beta)$ & $E W(\AA)$ & $F(\lambda) / F(\mathrm{H} \beta)$ & $I(\lambda) / I(\mathrm{H} \beta)$ & $E W(\AA)$ \\
\hline 3727 [O II] & $0.968 \pm 0.004$ & $0.949 \pm 0.004$ & $88.2 \pm 0.3$ & $1.099 \pm 0.016$ & $1.085 \pm 0.016$ & $79.2 \pm 0.8$ \\
\hline 3750 H12 & $0.024 \pm 0.001$ & $0.060 \pm 0.004$ & $2.2 \pm 0.1$ & $0.007 \pm 0.004$ & $0.031 \pm 0.021$ & $0.5 \pm 0.3$ \\
\hline $3771 \mathrm{H} 11$ & $0.029 \pm 0.001$ & $0.064 \pm 0.003$ & $2.7 \pm 0.1$ & $0.030 \pm 0.004$ & $0.053 \pm 0.009$ & $2.3 \pm 0.3$ \\
\hline 3798 H10 & $0.038 \pm 0.001$ & $0.073 \pm 0.003$ & $3.5 \pm 0.1$ & $0.045 \pm 0.005$ & $0.067 \pm 0.009$ & $3.4 \pm 0.4$ \\
\hline 3835 H9 & $0.057 \pm 0.001$ & $0.091 \pm 0.003$ & $5.3 \pm 0.1$ & $0.055 \pm 0.005$ & $0.078 \pm 0.008$ & $4.2 \pm 0.4$ \\
\hline 3868 [Ne III] & $0.262 \pm 0.002$ & $0.257 \pm 0.002$ & $25.2 \pm 0.2$ & $0.241 \pm 0.007$ & $0.238 \pm 0.007$ & $18.4 \pm 0.5$ \\
\hline 3889 H8 + He I & $0.166 \pm 0.002$ & $0.196 \pm 0.002$ & $16.4 \pm 0.2$ & $0.151 \pm 0.006$ & $0.172 \pm 0.008$ & $11.6 \pm 0.4$ \\
\hline $3968[\mathrm{Ne}$ III $]+\mathrm{H} 7$ & $0.227 \pm 0.002$ & $0.255 \pm 0.003$ & $23.0 \pm 0.2$ & $0.217 \pm 0.007$ & $0.236 \pm 0.008$ & $17.2 \pm 0.5$ \\
\hline $4026 \mathrm{He} \mathrm{I}$ & $0.013 \pm 0.001$ & $0.013 \pm 0.001$ & $1.3 \pm 0.1$ & $\ldots$ & $\ldots$ & $\ldots$ \\
\hline $4068[\mathrm{~S}$ II $]$ & $0.008 \pm 0.001$ & $0.008 \pm 0.001$ & $0.8 \pm 0.1$ & $\ldots$ & $\ldots$ & $\ldots$ \\
\hline $4101 \mathrm{H} \delta$ & $0.245 \pm 0.002$ & $0.269 \pm 0.002$ & $27.6 \pm 0.2$ & $0.236 \pm 0.007$ & $0.253 \pm 0.008$ & $19.9 \pm 0.5$ \\
\hline $4340 \mathrm{H} \gamma$ & $0.452 \pm 0.002$ & $0.469 \pm 0.003$ & $56.9 \pm 0.3$ & $0.446 \pm 0.009$ & $0.458 \pm 0.010$ & $42.9 \pm 0.7$ \\
\hline 4363 [O III] & $0.085 \pm 0.001$ & $0.084 \pm 0.001$ & $10.8 \pm 0.2$ & $0.071 \pm 0.005$ & $0.070 \pm 0.005$ & $6.9 \pm 0.5$ \\
\hline $4388 \mathrm{He} \mathrm{I}$ & $0.004 \pm 0.001$ & $0.004 \pm 0.001$ & $0.5 \pm 0.2$ & $\ldots$ & $\ldots$ & $\ldots$ \\
\hline $4471 \mathrm{He} \mathrm{I}$ & $0.034 \pm 0.001$ & $0.033 \pm 0.001$ & $4.5 \pm 0.2$ & $0.034 \pm 0.004$ & $0.034 \pm 0.004$ & $3.6 \pm 0.4$ \\
\hline 4658 [Fe III] & $0.006 \pm 0.001$ & $0.006 \pm 0.001$ & $1.0 \pm 0.1$ & $\ldots$ & $\ldots$ & $\ldots$ \\
\hline $4686 \mathrm{He}$ II & $0.023 \pm 0.001$ & $0.023 \pm 0.001$ & $3.5 \pm 0.2$ & $\ldots$ & $\ldots$ & $\ldots$ \\
\hline $4713[\mathrm{Ar}$ IV $]+\mathrm{He} \mathrm{I}$ & $0.011 \pm 0.001$ & $0.011 \pm 0.001$ & $1.7 \pm 0.2$ & $\ldots$ & $\ldots$ & $\ldots$ \\
\hline $4740[$ Ar IV $]$ & $0.005 \pm 0.001$ & $0.005 \pm 0.001$ & $0.8 \pm 0.2$ & $\ldots$ & $\ldots$ & $\ldots$ \\
\hline $4861 \mathrm{H} \beta$ & $1.000 \pm 0.004$ & $1.000 \pm 0.004$ & $166.2 \pm 0.5$ & $1.000 \pm 0.014$ & $1.000 \pm 0.014$ & $133.8 \pm 1.3$ \\
\hline $4922 \mathrm{He} \mathrm{I}$ & $0.008 \pm 0.001$ & $0.008 \pm 0.001$ & $1.4 \pm 0.2$ & $0.012 \pm 0.004$ & $0.012 \pm 0.004$ & $1.7 \pm 0.5$ \\
\hline 4959 [O III] & $1.203 \pm 0.005$ & $1.179 \pm 0.005$ & $214.2 \pm 0.6$ & $1.082 \pm 0.015$ & $1.068 \pm 0.015$ & $156.0 \pm 1.5$ \\
\hline 5007 [O III] & $3.613 \pm 0.012$ & $3.542 \pm 0.012$ & $663.0 \pm 0.9$ & $3.210 \pm 0.036$ & $3.169 \pm 0.036$ & $473.6 \pm 2.4$ \\
\hline 5199 [N I] & $0.005 \pm 0.001$ & $0.005 \pm 0.001$ & $1.0 \pm 0.2$ & $\ldots$ & $\ldots$ & $\ldots$ \\
\hline $5271[\mathrm{Fe}$ III] & $0.003 \pm 0.001$ & $0.003 \pm 0.001$ & $0.7 \pm 0.2$ & $\ldots$ & $\ldots$ & $\ldots$ \\
\hline $5518[\mathrm{Cl} \mathrm{III}]$ & $0.003 \pm 0.001$ & $0.003 \pm 0.001$ & $0.8 \pm 0.2$ & $\ldots$ & $\ldots$ & $\ldots$ \\
\hline $5538[\mathrm{Cl} \mathrm{III}]$ & $0.002 \pm 0.001$ & $0.002 \pm 0.001$ & $0.4 \pm 0.2$ & $\ldots$ & $\ldots$ & $\ldots$ \\
\hline 5876 He I & $0.100 \pm 0.001$ & $0.098 \pm 0.001$ & $28.8 \pm 0.3$ & $0.107 \pm 0.004$ & $0.106 \pm 0.004$ & $26.2 \pm 1.0$ \\
\hline 6300 [O I] & $0.022 \pm 0.001$ & $0.022 \pm 0.001$ & $8.0 \pm 0.3$ & $0.032 \pm 0.003$ & $0.032 \pm 0.003$ & $9.8 \pm 0.9$ \\
\hline 6312 [S III] & $0.015 \pm 0.001$ & $0.015 \pm 0.001$ & $5.3 \pm 0.2$ & $0.012 \pm 0.002$ & $0.011 \pm 0.002$ & $3.5 \pm 0.7$ \\
\hline 6364 [O I] & $0.008 \pm 0.001$ & $0.008 \pm 0.001$ & $2.7 \pm 0.2$ & $0.011 \pm 0.002$ & $0.011 \pm 0.002$ & $3.4 \pm 0.7$ \\
\hline $6563 \mathrm{H} \alpha$ & $2.714 \pm 0.009$ & $2.670 \pm 0.010$ & $997.9 \pm 1.7$ & $2.810 \pm 0.031$ & $2.780 \pm 0.034$ & $871.7 \pm 4.5$ \\
\hline 6584 [N II] & $0.033 \pm 0.001$ & $0.032 \pm 0.001$ & $11.1 \pm 0.3$ & $0.042 \pm 0.003$ & $0.042 \pm 0.003$ & $13.2 \pm 1.0$ \\
\hline $6678 \mathrm{He}$ I & $0.029 \pm 0.001$ & $0.028 \pm 0.001$ & $11.1 \pm 0.3$ & $0.029 \pm 0.003$ & $0.028 \pm 0.003$ & $9.2 \pm 0.8$ \\
\hline 6717 [S II] & $0.090 \pm 0.001$ & $0.088 \pm 0.001$ & $34.1 \pm 0.4$ & $0.107 \pm 0.004$ & $0.105 \pm 0.004$ & $34.3 \pm 1.2$ \\
\hline 6731 [S II] & $0.066 \pm 0.001$ & $0.065 \pm 0.001$ & $25.2 \pm 0.4$ & $0.080 \pm 0.004$ & $0.079 \pm 0.004$ & $25.9 \pm 1.1$ \\
\hline $7065 \mathrm{He} \mathrm{I}$ & $0.027 \pm 0.001$ & $0.026 \pm 0.001$ & $11.8 \pm 0.3$ & $0.027 \pm 0.002$ & $0.027 \pm 0.002$ & $8.4 \pm 0.9$ \\
\hline 7136 [Ar III] & $0.043 \pm 0.001$ & $0.042 \pm 0.001$ & $20.6 \pm 0.4$ & $0.048 \pm 0.003$ & $0.048 \pm 0.003$ & $21.0 \pm 1.3$ \\
\hline$C(\mathrm{H} \beta) \operatorname{dex}$ & & $0.000 \pm 0.004$ & & & $0.000 \pm 0.014$ & \\
\hline$F(\mathrm{H} \beta)^{\mathrm{a}}$ & & $4.54 \pm 0.01$ & & & $0.71 \pm 0.01$ & \\
\hline$E W($ abs $) \AA$ & & $3.4 \pm 0.1$ & & & $1.8 \pm 0.3$ & \\
\hline
\end{tabular}

${ }^{a}$ In units $10^{-14} \mathrm{erg} \mathrm{s}^{-1} \mathrm{~cm}^{-2}$.

$\mathrm{H} \alpha$ and $\mathrm{H} \beta$ emission lines are present in regions a1, a 3 and e 3 while $\mathrm{H} \gamma$ and $\mathrm{H} \delta$ absorption lines are detected in the spectra of all regions except for region e3. This allows us to derive the age of the stellar population using four methods, based on: (1) the time evolution of equivalent widths $(E W)$ of hydrogen emission lines, (2) the time evolution of $E W$ s of hydrogen absorption lines, (3) the comparison of the observed and theoretical spectral energy distributions, and (4) the broad-band colours. The requirement of consistency of the ages determined from the reddening-insensitive methods 1 and 2 and from the reddening-sensitive methods 3 and 4 allows to simultaneously 
Table 2. Element abundances in regions e1 and e2.

\begin{tabular}{|c|c|c|}
\hline Value & region e1 & region e2 \\
\hline$T_{\mathrm{e}}(\mathrm{O}$ III $)(\mathrm{K})$ & $16490 \pm 140$ & $15910 \pm 540$ \\
\hline$T_{\mathrm{e}}(\mathrm{O} \mathrm{II})(\mathrm{K})$ & $14430 \pm 110$ & $14170 \pm 460$ \\
\hline$T_{\mathrm{e}}(\mathrm{S}$ III $)(\mathrm{K})$ & $15380 \pm 110$ & $14900 \pm 450$ \\
\hline$N_{\mathrm{e}}(\mathrm{S} \mathrm{II})\left(\mathrm{cm}^{-3}\right)$ & $60 \pm 30$ & $90 \pm 80$ \\
\hline $\mathrm{O}^{+} / \mathrm{H}^{+}\left(\times 10^{5}\right)$ & $0.930 \pm 0.020$ & $1.127 \pm 0.101$ \\
\hline $\mathrm{O}^{+2} / \mathrm{H}^{+}\left(\times 10^{5}\right)$ & $3.071 \pm 0.063$ & $2.997 \pm 0.256$ \\
\hline $\mathrm{O}^{+3} / \mathrm{H}^{+}\left(\times 10^{6}\right)$ & $0.994 \pm 0.057$ & $\ldots$ \\
\hline $\mathrm{O} / \mathrm{H}\left(\times 10^{5}\right)$ & $4.100 \pm 0.067$ & $4.123 \pm 0.275$ \\
\hline $12+\log (\mathrm{O} / \mathrm{H})$ & $7.61 \pm 0.01$ & $7.62 \pm 0.03$ \\
\hline $\mathrm{N}^{+} / \mathrm{H}^{+}\left(\times 10^{7}\right)$ & $2.564 \pm 0.056$ & $3.460 \pm 0.311$ \\
\hline $\operatorname{ICF}(\mathrm{N})^{a}$ & 4.41 & 3.66 \\
\hline $\log (\mathrm{N} / \mathrm{O})$ & $-1.560 \pm 0.017$ & $-1.513 \pm 0.069$ \\
\hline $\mathrm{Ne}^{+2} / \mathrm{H}^{+}\left(\times 10^{5}\right)$ & $0.480 \pm 0.011$ & $0.491 \pm 0.045$ \\
\hline $\operatorname{ICF}(\mathrm{Ne})^{a}$ & 1.34 & 1.38 \\
\hline $\log (\mathrm{Ne} / \mathrm{O})$ & $-0.806 \pm 0.014$ & $-0.786 \pm 0.057$ \\
\hline $\mathrm{S}^{+} / \mathrm{H}^{+}\left(\times 10^{7}\right)$ & $1.618 \pm 0.025$ & $2.028 \pm 0.118$ \\
\hline $\mathrm{S}^{+2} / \mathrm{H}^{+}\left(\times 10^{7}\right)$ & $6.980 \pm 0.350$ & $5.942 \pm 1.335$ \\
\hline $\operatorname{ICF}(\mathrm{S})^{a}$ & 1.50 & 1.39 \\
\hline $\log (\mathrm{S} / \mathrm{O})$ & $-1.503 \pm 0.016$ & $-1.571 \pm 0.068$ \\
\hline $\mathrm{Cl}^{++} / \mathrm{H}^{+}\left(\times 10^{8}\right)$ & $1.017 \pm 0.223$ & $\ldots$ \\
\hline $\mathrm{ICF}(\mathrm{Cl})^{a}$ & 1.57 & $\ldots$ \\
\hline $\log (\mathrm{Cl} / \mathrm{O})$ & $-3.409 \pm 0.076$ & $\ldots$ \\
\hline $\mathrm{Ar}^{+} 2 / \mathrm{H}^{+}\left(\times 10^{7}\right)$ & $1.449 \pm 0.032$ & $1.743 \pm 0.130$ \\
\hline $\mathrm{Ar}^{+3} / \mathrm{H}^{+}\left(\times 10^{7}\right)$ & $0.608 \pm 0.119$ & $\ldots$ \\
\hline $\mathrm{ICF}(\mathrm{Ar})^{a}$ & 1.05 & 1.64 \\
\hline $\log (\mathrm{Ar} / \mathrm{O})$ & $-2.280 \pm 0.026$ & $-2.158 \pm 0.038$ \\
\hline $\mathrm{Fe}^{++} / \mathrm{H}^{+}\left(\times 10^{7}\right)$ & $1.320 \pm 0.200$ & $\ldots$ \\
\hline $\operatorname{ICF}(\mathrm{Fe})^{a}$ & 5.51 & $\ldots$ \\
\hline $\log (\mathrm{Fe} / \mathrm{O})$ & $-1.751 \pm 0.016$ & $\ldots$ \\
\hline$[\mathrm{O} / \mathrm{Fe}]^{b}$ & $0.331 \pm 0.029$ & $\ldots$ \\
\hline $\mathrm{He}^{+} / \mathrm{H}^{+}(\lambda 4471)$ & $0.071 \pm 0.002$ & $0.070 \pm 0.009$ \\
\hline $\mathrm{He}^{+} / \mathrm{H}^{+}(\lambda 5876)$ & $0.081 \pm 0.001$ & $0.083 \pm 0.004$ \\
\hline $\mathrm{He}^{+} / \mathrm{H}^{+}(\lambda 6678)$ & $0.082 \pm 0.002$ & $0.081 \pm 0.008$ \\
\hline \multicolumn{3}{|l|}{$\mathrm{He}^{+} / \mathrm{H}^{+}$} \\
\hline (weighted mean) & $0.080 \pm 0.001$ & $0.080 \pm 0.003$ \\
\hline $\mathrm{He}^{+2} / \mathrm{H}^{+}(\lambda 4686)$ & $0.002 \pm 0.000$ & $\ldots$ \\
\hline $\mathrm{He} / \mathrm{H}$ & $0.082 \pm 0.001$ & $0.080 \pm 0.003$ \\
\hline$Y$ & $0.246 \pm 0.003$ & $0.243 \pm 0.010$ \\
\hline
\end{tabular}

${ }^{a} \mathrm{ICF}$ is the ionization correction factor.

${ }^{b}[\mathrm{O} / \mathrm{Fe}] \equiv \log (\mathrm{O} / \mathrm{Fe})-\log (\mathrm{O} / \mathrm{Fe})_{\odot}$.

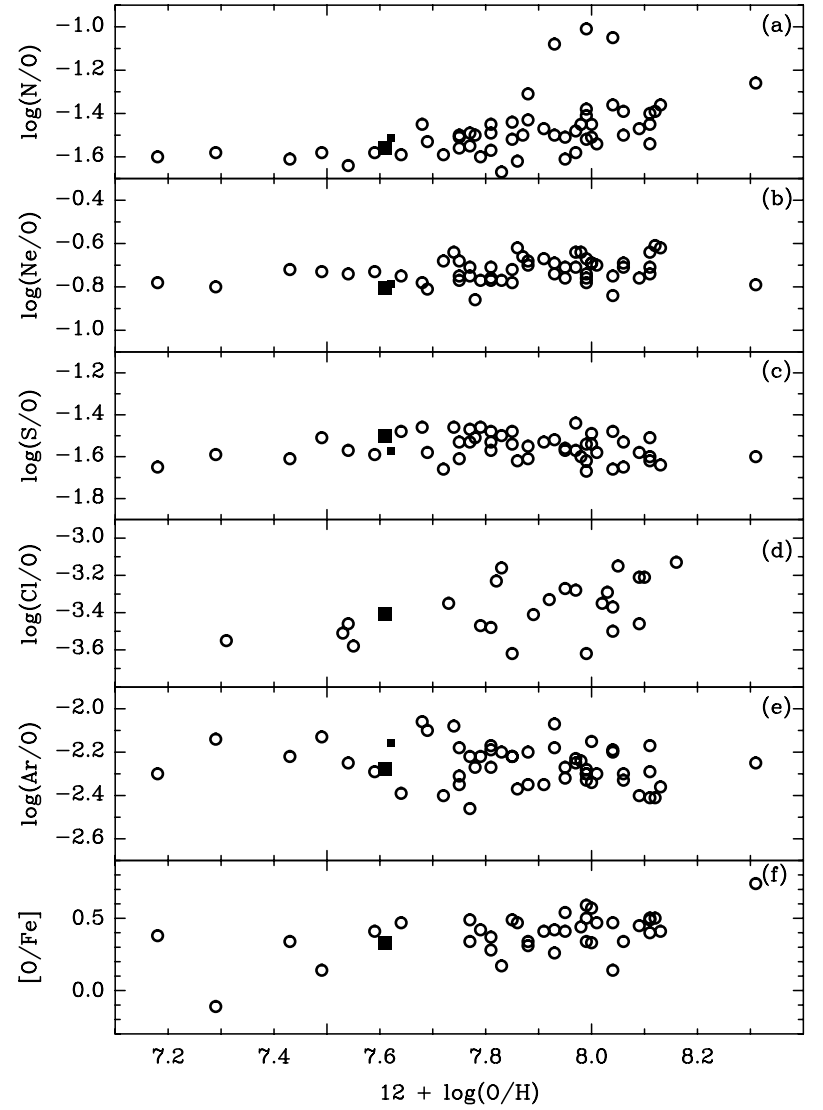

Fig. 5. Comparison of the elemental abundance ratios, obtained for the brightest $\mathrm{H}$ II regions e1 (large squares) and e2 (small squares) with data for other BCDs (open circles).

derive the extinction coefficient and constrain the SF history (Guseva et al. 2001, 2003a, 2003b).

We measured the fluxes and equivalent widths of the $\mathrm{H} \alpha$ and $\mathrm{H} \beta$ emission lines and the $\mathrm{H} \gamma$ and $\mathrm{H} \delta$ absorption lines in the spectra of the LSB regions, and list them in Tables 3 and 4. Because the $\mathrm{H} \beta$ emission line is narrower than the absorption line in these regions and does not fill the absorption component, its flux was measured using the continuum level at the bottom of the absorption line. This level was chosen by visually interpolating from the absorption line wings to the center of the line.

The extinction coefficient $C(\mathrm{H} \beta)$ in those regions is derived from the $\mathrm{H} \alpha / \mathrm{H} \beta$ flux ratio. We adopt the theoretical recombination $\mathrm{H} \alpha / \mathrm{H} \beta$ flux ratio of 2.8 , which is typical for hot lowmetallicity $\mathrm{H}$ II regions. No correction for the absorption line equivalent widths has been made. The extinction coefficients $C(\mathrm{H} \beta)$ are shown in Table 3.

Hydrogen absorption lines are seen in the spectra of all regions labeled in Fig. 1 except for the brightest $\mathrm{H}$ II regions e1 and $\mathrm{e} 2$ and the LSB region e3. The blue part of the spectrum of region a1 with hydrogen absorption and emission lines is shown in Fig. 6. Table 4 lists the equivalent widths with their errors of the $\mathrm{H} \gamma$ and $\mathrm{H} \delta$ absorption lines measured in the wavelength intervals or "windows" of Bica \& Alloin (1986). The errors include the errors in the fitting of line profiles with Gaussians and the noise dispersion in the continuum. A careful 
Table 3. Fluxes, equivalent widths of the $\mathrm{H} \alpha$ and $\mathrm{H} \beta$ emission lines and the extinction coefficients $C(\mathrm{H} \beta)$ in the LSB regions.

\begin{tabular}{lcccccccc}
\hline \hline Telescope & Region & Distance $^{a}$ & Aperture $^{b}$ & $F(\mathrm{H} \alpha)^{c}$ & $F(\mathrm{H} \beta)^{c}$ & $E W(\mathrm{H} \alpha)^{d}$ & $E W(\mathrm{H} \beta)^{d}$ & $C(\mathrm{H} \beta)$ \\
\hline MMT $^{e}$ & $a 1$ & 10.7 & $1.5 \times 3.4$ & $3.53 \pm 0.14$ & $1.25 \pm 0.12$ & $12.30 \pm 0.75$ & $2.72 \pm 0.24$ & $0.0 \pm 0.03$ \\
& $a 2$ & 29.5 & $1.5 \times 13.2$ & $\ldots$ & $\ldots$ & $\ldots$ & $\ldots$ & $\ldots$ \\
\hline $4 \mathrm{~m}^{f}$ & $a 3$ & 12.6 & $2.0 \times 7.6$ & $4.88 \pm 0.39$ & $1.88 \pm 0.29$ & $32.81 \pm 2.32$ & $7.72 \pm 1.01$ & $0.0 \pm 0.04$ \\
& $e 3$ & 18.4 & $2.0 \times 2.8$ & $3.69 \pm 0.17$ & $1.19 \pm 0.14$ & $109.80 \pm 1.41$ & $16.57 \pm 0.69$ & $0.04 \pm 0.06$ \\
& $a 4$ & 22.6 & $2.0 \times 2.8$ & $\ldots$ & $\ldots$ & $\ldots$ & $\ldots$ & $\ldots$ \\
\hline
\end{tabular}

${ }^{a}$ Distance in arcsec from the brightest $\mathrm{H}$ II region e1.

${ }^{b}$ Aperture size $x \times y$, where $x$ is the slit width and $y$ the size along the slit in arcsec.

${ }^{c}$ In units $10^{-16} \mathrm{erg} \mathrm{s}^{-1} \mathrm{~cm}^{-2}$.

${ }^{d}$ In $\AA$.

${ }^{e}$ Slit orientation with position angle $\mathrm{PA}=22^{\circ}$.

${ }^{f}$ Slit orientation with position angle $\mathrm{PA}=48^{\circ}$.

Table 4. Equivalent widths of the $\mathrm{H} \gamma$ and $\mathrm{H} \delta$ absorption lines in the LSB regions.

\begin{tabular}{lcclcc}
\hline \hline Telescope & Region & Distance $^{a}$ & Aperture $^{b}$ & $E W(\mathrm{H} \delta)^{c}$ & $E W(\mathrm{H} \gamma)^{c}$ \\
\hline MMT $^{d}$ & $a 1$ & 10.7 & $1.5 \times 3.4$ & $-5.53 \pm 0.22$ & $-4.92 \pm 0.23$ \\
& $a 2$ & 29.5 & $1.5 \times 13.2$ & $-8.89 \pm 0.53$ & $-6.07 \pm 0.41$ \\
\hline \multirow{2}{*}{$4 \mathrm{~m}^{e}$} & $a 3$ & 12.6 & $2.0 \times 7.6$ & $-8.28 \pm 0.50$ & $-5.71 \pm 0.60$ \\
& $a 4$ & 22.6 & $2.0 \times 2.8$ & $-9.06 \pm 0.63$ & $\ldots$ \\
\hline
\end{tabular}

${ }^{a}$ Distance in arcsec from the brightest $\mathrm{H}$ II region e1.

${ }^{b}$ Aperture size $x \times y$, where $x$ is the slit width and $y$ the size along the slit in arcsec.

${ }^{c}$ In $\AA$.

${ }^{d}$ Slit orientation with position angle $\mathrm{PA}=22^{\circ}$.

${ }^{e}$ Slit orientation with position angle $\mathrm{PA}=48^{\circ}$.

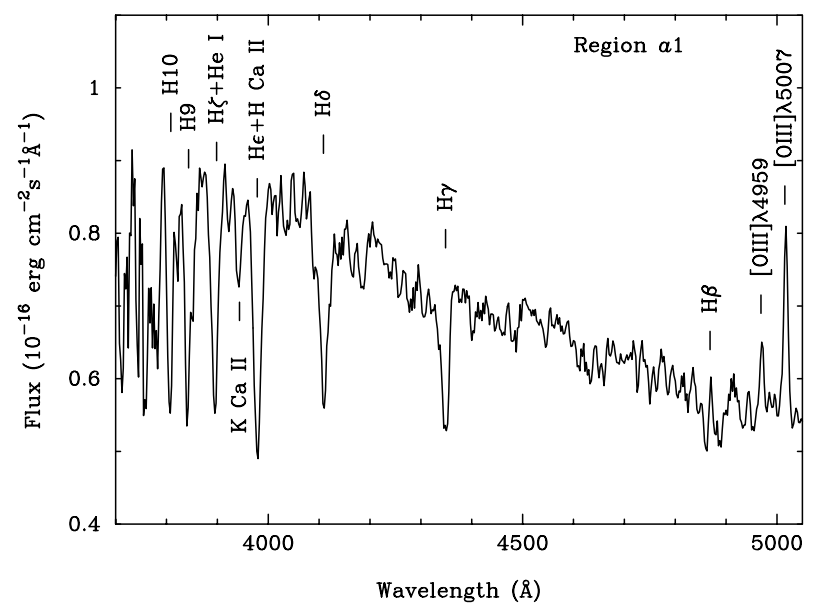

Fig. 6. The blue part of the MMT spectrum of region a1 with labeled emission and absorption lines.

placement of the continuum level is very important for deriving accurate $E W \mathrm{~s}$. For this purpose, we choose points in the spectrum free of nebular and stellar lines, which were then fitted by cubic splines. The uncertainties were estimated from several different measurements of the equivalent widths of hydrogen absorption lines with independent continuum fittings. They are of the same order as the errors in Table 4.

\subsection{Age calibration}

The calibration of the age of stellar populations using the equivalent widths of the $\mathrm{H} \alpha$ and $\mathrm{H} \beta$ nebular emission lines, those of the $\mathrm{H} \gamma$ and $\mathrm{H} \delta$ stellar absorption lines and the spectral energy distributions is discussed in detail in Guseva et al. (2001, 2003a, 2003b). Here we only briefly describe these calibrations.

\subsubsection{Balmer emission lines}

The temporal evolution of the $\mathrm{H} \alpha$ and $\mathrm{H} \beta$ emission line equivalent widths depends on the star formation history. We consider here the two limiting cases of instantaneous burst and continuous star formation models. The equivalent widths for the instantaneous burst model with a heavy element mass fraction $Z_{\odot} / 20$ are calculated using the galactic evolution code PEGASE. 2 (Fioc \& Rocca-Volmerange 1997). The dependence of the $\mathrm{H} \alpha$ emission line equivalent width on time is shown in Fig. 6a of Guseva et al. (2003b) by the thick solid line. These models are appropriate for regions e 1 and e 2 with strong emission lines. The equivalent widths of Balmer emission lines in region e $1(E W(\mathrm{H} \alpha)=998 \AA$ and $E W(\mathrm{H} \beta)=166 \AA)$ and region $\mathrm{e} 2(E W(\mathrm{H} \alpha)=872 \AA$ and $E W(\mathrm{H} \beta)=134 \AA)$ correspond to an instantaneous burst age of $4 \mathrm{Myr}$.

However, for the LSB regions, models with continuous star formation are more appropriate. For these models we adopt 
a constant star formation rate (SFR) within the time interval from $t_{\mathrm{i}}$ when star formation starts to $t_{\mathrm{f}}$ when it stops. Time is zero now and increases to the past. The equivalent widths of hydrogen emission lines and SEDs for a set of instantaneous burst models (Fioc \& Rocca-Volmerange 1997) are used to calculate the temporal evolution of $E W$ s for continuous SF with a constant SFR. The temporal dependence of the equivalent widths of the $\mathrm{H} \alpha$ emission line is shown in Fig. 6a of Guseva et al. (2003b) for different $t_{\mathrm{i}}$ and $t_{\mathrm{f}}$.

\subsubsection{Stellar Balmer absorption lines}

Another way to derive the age of a stellar population is to use the relation between the $\mathrm{H} \delta$ and $\mathrm{H} \gamma$ absorption line equivalent widths and age, derived by González Delgado et al. (1999). Their instantaneous burst models predict a steady increase of the equivalent widths with age from 1 Myr to 1 Gyr. However, they did not extend the calculations for ages $\gtrsim 1$ Gyr when the equivalent widths of the absorption lines decrease with age (Bica \& Alloin 1986). Hence, each value of the hydrogen absorption line equivalent width corresponds to two values of the age, $\lesssim 1$ Gyr and $\gtrsim 1$ Gyr. This ambiguity can be resolved with the use of other age constraints discussed in this paper.

Furthermore, the models by González Delgado et al. (1999) probably overestimate the equivalent widths of the absorption lines at ages $\sim 1$ Gyr (Guseva et al. 2003b). Therefore, in the age range from $1 \mathrm{Myr}$ to $16.5 \mathrm{Gyr}$ instead of the calibration by González Delgado et al. (1999) we use an empirical calibration of the hydrogen absorption line equivalent widths versus age by Bica \& Alloin (1986). This calibration is based on the integrated spectra of 63 open and globular stellar clusters with known ages, metallicities and reddenings which can be used as templates for stellar populations formed in an instantaneous burst. For consistency we use the same wavelength intervals or "windows" for $\mathrm{H} \gamma$ and $\mathrm{H} \delta$ flux measurements as Bica \& Alloin (1986) ( $\lambda 4318-4364 \AA$ and $\lambda 4082-4124 \AA$, respectively).

The behaviour of the empirical $\mathrm{H} \delta$ absorption line equivalent width with the age for an instantaneous burst (Bica \& Alloin 1986) is shown in Fig. 6b of Guseva et al. (2003b) by the thick solid line.

The temporal evolution of the $\mathrm{H} \gamma$ and $\mathrm{H} \delta$ absorption line equivalent widths in the case of continuous SF is calculated similarly to that of the $\mathrm{H} \alpha$ and $\mathrm{H} \beta$ emission line equivalent widths described in Sect. 5.1.1. More specifically, we use the empirical equivalent widths of hydrogen absorption lines (Bica \& Alloin 1986) and SEDs for instantaneous bursts (Fioc \& Rocca-Volmerange 1997) to calculate the temporal evolution of $E W \mathrm{~s}$ in the case of continuous SF with constant SFR. The results are shown in Fig. 6b of Guseva et al. (2003b) for SF with different $t_{\mathrm{i}}$ and $t_{\mathrm{f}}$.

\subsubsection{Spectral energy distribution}

The shape of the spectrum reflects the properties of the stellar population. However, it is also dependent on the reddening. A precise determination of the extinction can be done only for the two brightest $\mathrm{H}$ II regions $\mathrm{e} 1$ and $\mathrm{e} 2$ which possess many strong hydrogen emission lines (Table 1 ). We derived an extinction coefficient $C(\mathrm{H} \beta)=0$ in these regions. In the LSB regions a1, a3 and e3, only $\mathrm{H} \alpha$ and $\mathrm{H} \beta$ emission lines are present. The extinction coefficients obtained from the $\mathrm{H} \alpha / \mathrm{H} \beta$ flux ratio in these regions are small (Table 3 ). However, they are more uncertain as compared to the ones in regions e 1 and e 2 because of the weakness of the emission lines and significant contribution of the stellar absorption lines. $\mathrm{H} \alpha$ and $\mathrm{H} \beta$ emission lines are not detected in the other LSB regions. Therefore, the observed SED cannot directly give information on the age, but should be used together with the methods discussed in Sects. 5.1.1 and 5.1.2 for simultaneous determination of the age and interstellar extinction.

We used the galactic evolution code PEGASE.2 (Fioc \& Rocca-Volmerange 1997) to produce a grid of theoretical SEDs for an instantaneous burst of star formation with ages ranging between 0 and $10 \mathrm{Gyr}$, and a heavy element mass fraction of $Z=Z_{\odot} / 20$. The SEDs for continuous SF in the time interval between $t_{\mathrm{i}}$ ago and $t_{\mathrm{f}}$ ago are derived by integration of instantaneous burst SEDs.

\subsection{Ages of the stellar populations in the LSB regions}

In this section we derive self-consistently the ages of the stellar populations in the LSB regions using: 1) the equivalent widths of emission lines, 2) the equivalent widths of absorption lines, 3) the SEDs and 4) the colours. For this we adopt a continuous SF scenario with constant or variable SFR. In the latter case we consider a simplified scenario with two time intervals of SF, which we call young and old, with different SFRs. To quantify the difference in SFRs we introduce the parameter $b=\mathrm{SFR}$ (young)/SFR(old). Because of the noisy spectrum of region $\mathrm{e} 3$ and the significant contamination by nebular emission we were not able to measure equivalent widths of absorption lines in that region. Therefore, we exclude it from the analysis. For the remaining LSB regions, the model predictions providing the best fits to the observed SEDs are shown in Table 5. For each region we show the age range of one or two SF episodes, the parameter $b$, the mass ratio $M_{\mathrm{y}} / M_{\mathrm{o}}$ of the young-to-old populations, the model equivalent widths of hydrogen lines, the extinction coefficients $C(\mathrm{H} \beta)$ derived from the best match between observed and calculated SEDs, and the intrinsic and reddened $(V-I)$ colours (see Sect. 5.2.1). We use the relation $E(V-I)=0.891 C(\mathrm{H} \beta)$ (e.g., Aller 1984) to correct for reddening. Our young and old stellar populations in Table 5 include stars with ages not older than $250 \mathrm{Myr}$ $(\log t=8.4)$ and not younger than $160 \mathrm{Myr}(\log t=8.2)$, respectively. These definitions differ from those conventionally used. In fact, the old stellar population in our case includes not only several Gyr old stars but also intermediate-age stars with age $\lesssim 1$ Gyr. Negative $E W$ s denote absorption lines, positive $E W \mathrm{~s}$ refer to emission lines. Models with highlighted $E W \mathrm{~s}$ are those in which the equivalent widths are not reproduced well despite a good fit of the observed SEDs (compare model $E W \mathrm{~s}$ in Table 5 with observed $E W \mathrm{~s}$ in Tables 3 and 4).

We show in Fig. 7 the observed and predicted SEDs in the outer LSB region a2 (MMT observations) with no emission 

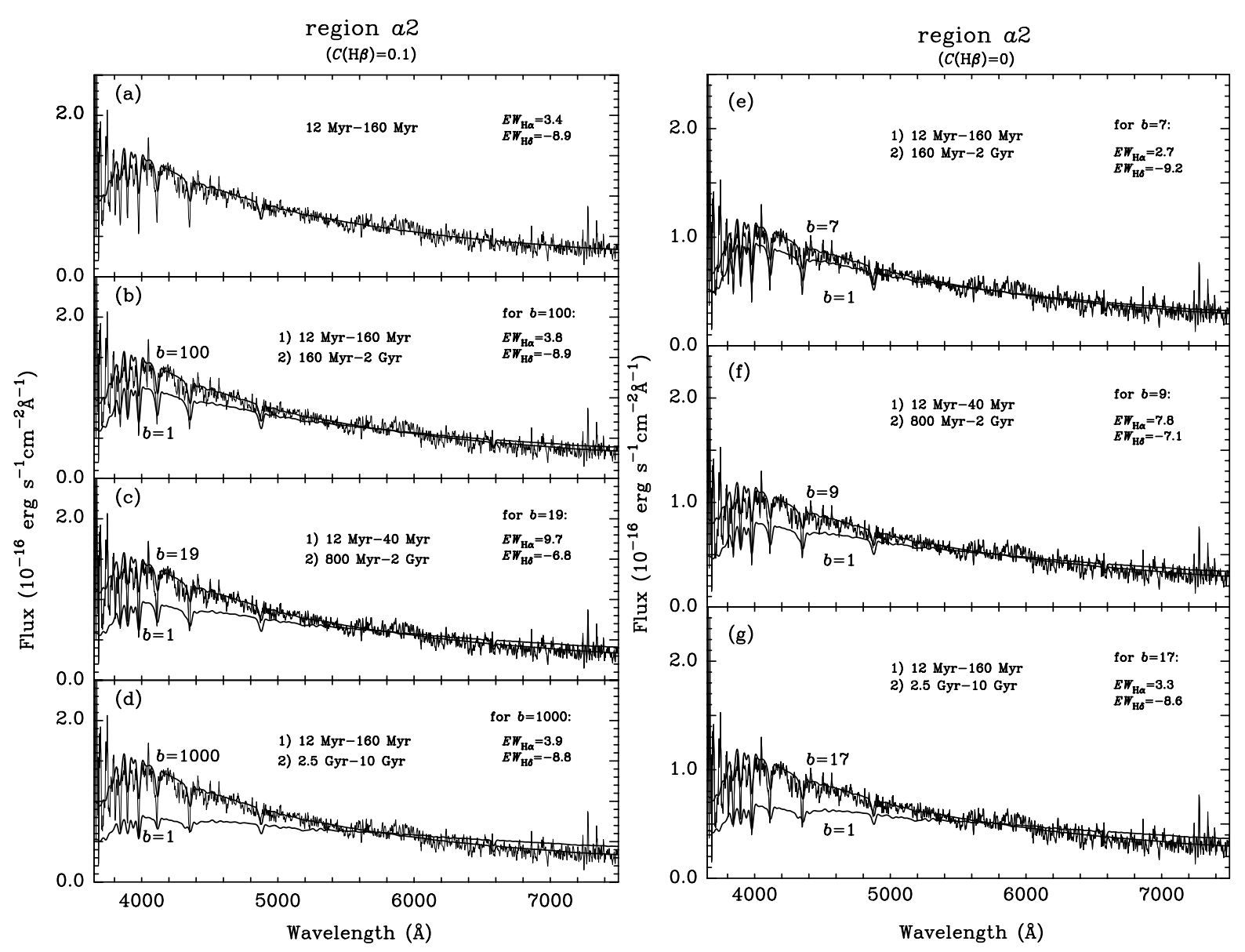

Fig. 7. Spectra of region $a 2$ on which synthetic SEDs are superposed. Synthetic SEDs are calculated for stellar populations forming continuously during one a) or two (b)-g)) time intervals. In the case of two intervals the SFR is constant within each interval but varies from one interval to another one by a factor $b=\operatorname{SFR}$ (young)/SFR(old). The spectra in left panel are corrected for interstellar extinction with $C(\mathrm{H} \beta)=0.1$. The extinction coefficient $C(\mathrm{H} \beta)$ is set to 0 in right panel. Time intervals, parameters $b$ and predicted $E W \mathrm{~s}$ of the $\mathrm{H} \alpha$ emission and $\mathrm{H} \delta$ absorption lines are indicated (see explanations in Sect. 5.2).

features. The $\mathrm{H} \gamma$ and $\mathrm{H} \delta$ absorption lines in this region as well as in the other outer region a4 (4 m telescope observations) are not contaminated by nebular emission from young stellar populations. Hence, the absorption line equivalent widths in regions a 2 and $\mathrm{a} 4$ are more accurate than in other LSB regions (Table 4).

We consider several SF histories and vary extinction in the LSB regions to put constraints on the age of their stellar populations. Figure 7 shows the spectra of region a 2 on which are superposed the synthetic SEDs with different SF histories which best fit it.

We have assumed two extinction coefficients: $C(\mathrm{H} \beta)=0.1$, derived by Thuan et al. (1999), and corresponding to $A(V) \sim$ 0.2 , and $C(\mathrm{H} \beta)=0$.

The model SEDs, adopting $C(\mathrm{H} \beta)=0.1$ are shown in Fig. 7, left panel. The observed properties of region a 2 can be reproduced by a single young stellar population with age between 12 Myr and $160 \mathrm{Myr}$, if a small amount of extinction is assumed (Fig. 7a). There is no need to invoke an older stellar population which, if present, has to be much smaller in mass than the young stellar population. We note that there is no ongoing $\mathrm{SF}$ in this region. The most recent $\mathrm{SF}$ in region a2 stopped at time $\gtrsim 12 \mathrm{Myr}$ ago, otherwise its spectrum would have shown a detectable $\mathrm{H} \alpha$ emission line (Fig. 6a in Guseva et al. 2003b).

Next we consider SF scenarios with a varying SFR which include older stellar populations with an age of up to $2 \mathrm{Gyr}$ (Fig. 7b-7c) and 10 Gyr (Fig. 7d). But even in these cases, the young population completely dominates the light and mass of region a2 (Table 5).

An upper limit to the age of region a 2 can be obtained by assuming no extinction, i.e. $C(\mathrm{H} \beta)=0$ (Fig. 7, right panel). Then the observed SED cannot be reproduced by a synthetic SED with stars forming between $12 \mathrm{Myr}$ and $160 \mathrm{Myr}$ ago, as the latter is too blue. However, by varying the parameter $b$, the observed SED can be reproduced by SEDs of stellar populations with other SF scenarios. In Figs. 7e-g, we show the best fits labeled by the adopted value of $b$. We exclude the models shown in Figs. 7c and $\mathrm{f}$ because they do not fit the observed equivalent widths of $\mathrm{H} \alpha$ and $\mathrm{H} \delta$. The remaining two models with $C(\mathrm{H} \beta)=0$ satisfy all observational constraints. In these models, the SFRs for the young population are respectively 7 times (Fig. 7e) and 17 times (Fig. 7g) larger than SFRs for the old population. These ratios are, however, significantly smaller than the corresponding parameters $b$ in the case with 


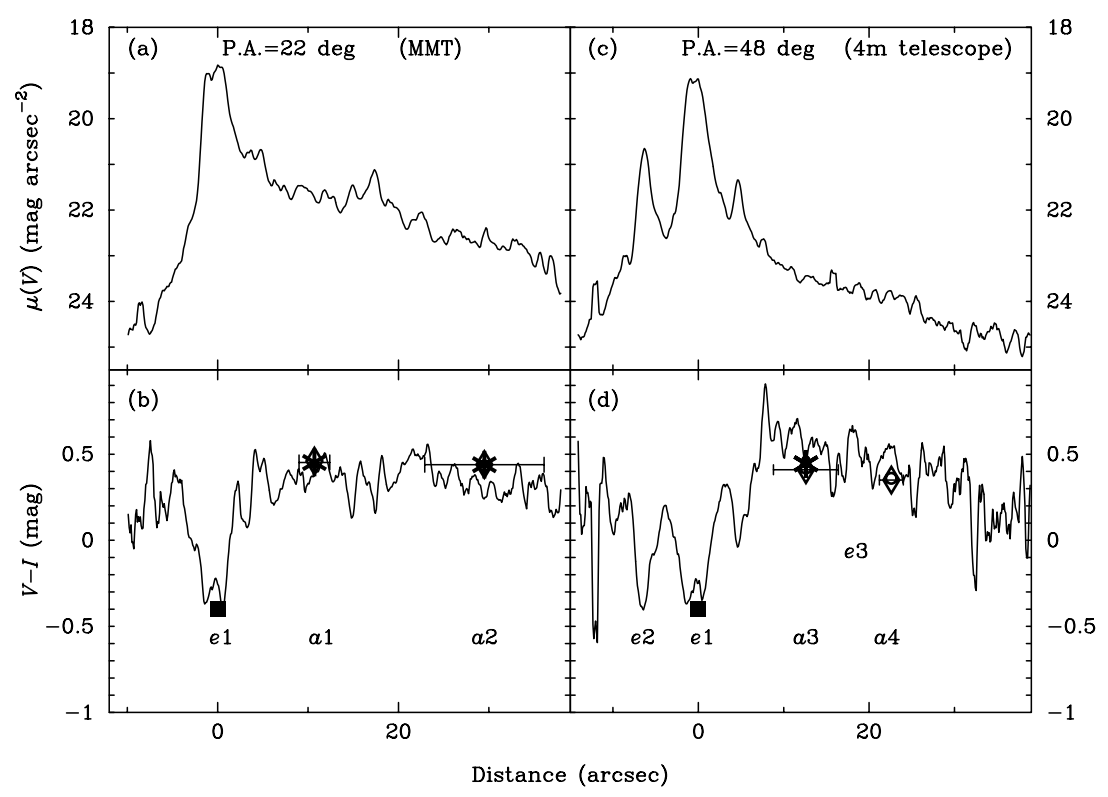

Fig. 8. a) $V$ surface brightness distribution along the slit oriented at position angle $\mathrm{PA}=22^{\circ}$. The origin is set at the brightest $\mathrm{H}$ II region e1. b) $(V-I)$ colour distribution along the slit with position angle $\mathrm{PA}=22^{\circ}$. c) $V$ surface brightness distribution along the slit oriented at position angle $\mathrm{PA}=48^{\circ}$. The origin is set at the brightest $\mathrm{H}$ II region e1. d) $(V-I)$ colour distribution along the slit with position angle PA $=48^{\circ}$. The locations of different regions are labeled in b) and d). Filled squares show the total modeled colours (stellar plus gaseous emission) for region e1. Other symbols show the colours predicted for the LSB regions by the models considered (Table 5).

$C(\mathrm{H} \beta)=0.1$. The relative mass fraction of the young stellar population is therefore smaller in the extinction-free case, being $M_{\mathrm{y}} / M_{\mathrm{o}}=0.6$ (Fig. 7e) and 0.3 (Fig. $7 \mathrm{~g}$ ), respectively. Therefore, the presence of a 2 Gyr old or even 10 Gyr old population is not excluded in region a2, which would however not dominate the optical emission. If the extinction is non-zero, which is likely the case, then there is no need to invoke a significant old population to explain the observed properties of region a2. It is likely that for other regions, a1 and a3 (Table 5), the extinction is even larger.

Similar consideration for region a4 shows that the mass fraction of the old stellar population is small even in the absence of extinction. In any case, the old stellar population, if present does not contribute significantly to the luminosity. All the observed properties of this region can be reproduced with a young stellar population formed between $12 \mathrm{Myr}$ and $160 \mathrm{Myr}$ ago. However, the presence of an older stellar population cannot be excluded in that case, but one needs to increase the parameter $b$ to match the observations (Table 5).

If some extinction is present in region a4, then our age upper limits will decrease. There is some hint that the extinction may increase with decreasing distance to the brightest $\mathrm{H}$ II region e1. Indeed, by considering models which include also a 10 Gyr old population and $C(\mathrm{H} \beta)=0$, we find from Table 5 that the closer a region is to the brightest part of the galaxy (region a1 compared to region a2, region a3 compared to region a4), the smaller is the relative mass $M_{\mathrm{y}} / M_{\mathrm{o}}$ of the young stellar population. This is in contrast to the trend found in BCDs where the relative mass of the young stellar population decreases outwards. Hence, we conclude, that the extinction in SBS $1415+437$ is larger in the inner brighter regions.
In summary, our spectroscopic analysis of the LSB regions favors a relatively young luminosity-weighted age of the stars populating those regions. A model with a constant SFR continuing from 10 Gyr ago until now is definitely excluded. An old population $\gtrsim 2$ Gyr could be present only in models with very specific SF scenarios, with a very low SF activity during the first 2-10 Gyr period, a very high star formation rate during the last $\sim(10-200)$ Myr, and a quiescent period in between. If, however, low SF activity has occurred in the period between 200 Myr and 2 Gyr ago, then there is no need to invoke a stellar population with age $\gtrsim 2 \mathrm{Gyr}$, and all the spectroscopic properties of the LSB regions can be explained by only young and intermediate-age stellar populations.

\subsubsection{Age from the colour distributions}

From the HST images, we derived $V$ and $I$ surface brightness and colour distributions for the regions covered by the spectroscopic observations with the MMT (Figs. 8a and b) and the KPNO $4 \mathrm{~m}$ telescope (Figs. 8c and d). Note the trend for the $(V-I)$ colour of the LSB component to decrease with increasing distance from region e1, especially for the KPNO $4 \mathrm{~m}$ data (Fig. 8d). This is again suggestive of larger extinction in the brighter regions. We compare the observed colours with predictions from our population synthesis modeling. The results of this comparison are shown in Figs. $8 \mathrm{~b}$ and $\mathrm{d}$. The predicted colours, obtained from convolving the theoretical SEDs with the appropriate filter bandpasses, are shown by different symbols. The transmission curves for the Johnson $V$ and Cousins $I$ bands are taken from Bessell (1990). The zero points are from Bessell et al. (1998). 
Table 5. Predicted equivalent widths of emission and absorption lines, extinction coefficients and $(V-I)$ colours from models of continuous star formation.

\begin{tabular}{|c|c|c|c|c|c|c|c|c|c|c|c|c|}
\hline & \multirow{3}{*}{ Region } & \multicolumn{2}{|c|}{ Age range $^{a}$} & \multirow{3}{*}{$b^{b}$} & \multirow{3}{*}{$M_{\mathrm{y}} / M_{\mathrm{o}}$} & \multicolumn{4}{|c|}{ modeled $E W$} & \multirow{3}{*}{$C(\mathrm{H} \beta)$} & \multicolumn{2}{|c|}{ modeled $(V-I)^{d}$} \\
\hline & & young & old & & & $\mathrm{H} \delta^{c}$ & $\mathrm{H} \gamma^{c}$ & $\mathrm{H} \beta^{c}$ & $\mathrm{H} \alpha^{c}$ & & intrinsic & reddened \\
\hline & & $t_{\mathrm{f}}-t_{\mathrm{i}}$ & $t_{\mathrm{f}}-t_{\mathrm{i}}$ & & & & & & & & & \\
\hline \multirow[t]{11}{*}{$\mathrm{MMT}^{e}$} & \multirow[t]{4}{*}{ a1 } & \multicolumn{2}{|c|}{$7.1-7.5$} & $\ldots$ & $\ldots$ & -6.3 & -5.2 & 2.2 & 13.8 & 0.27 & 0.211 & 0.452 \\
\hline & & $7.05-7.5$ & $9.4-10$ & 24 & 0.07 & -6.0 & -5.1 & 2.5 & 13.2 & 0.00 & 0.456 & 0.456 \\
\hline & & $7.1-7.5$ & $9.4-10$ & 66 & 0.18 & -6.2 & -5.2 & 2.2 & 12.9 & 0.12 & 0.335 & 0.442 \\
\hline & & $7.11-7.5$ & $9.4-10$ & 400 & 1.00 & -6.2 & -5.2 & 1.9 & 13.6 & 0.22 & 0.242 & 0.438 \\
\hline & \multirow[t]{7}{*}{$\mathrm{a} 2$} & \multicolumn{2}{|c|}{$7.1-8.2$} & $\ldots$ & $\ldots$ & -8.9 & -7.9 & 0.6 & 3.4 & 0.10 & 0.350 & 0.439 \\
\hline & & $7.1-8.2$ & $8.2-9.3$ & 100 & 8.1 & -8.9 & -7.9 & 0.7 & 3.8 & 0.10 & 0.358 & 0.447 \\
\hline & & $7.1-7.6$ & $8.9-9.3$ & 19 & 0.5 & $-6.8^{g}$ & -5.6 & 1.7 & $9.7^{g}$ & 0.10 & 0.342 & 0.431 \\
\hline & & $7.1-8.2$ & $9.4-10$ & 1000 & 19.7 & -8.8 & -7.9 & 0.7 & 3.9 & 0.10 & 0.352 & 0.441 \\
\hline & & $7.1-8.2$ & $8.2-9.3$ & 7 & 0.6 & -9.2 & -7.9 & 0.5 & 2.7 & 0.00 & 0.440 & 0.440 \\
\hline & & $7.1-7.6$ & $8.9-9.3$ & 9 & 0.2 & $-7.1^{g}$ & -5.8 & 1.4 & $\mathbf{7 . 8}^{g}$ & 0.00 & 0.418 & 0.418 \\
\hline & & $7.1-8.2$ & $9.4-10$ & 17 & 0.3 & -8.6 & -7.7 & 0.6 & 3.3 & 0.00 & 0.441 & 0.441 \\
\hline \multirow[t]{9}{*}{ KPNO $4 \mathrm{~m}^{f}$} & \multirow[t]{5}{*}{ a3 } & \multicolumn{2}{|c|}{$6.7-8.4$} & $\ldots$ & $\ldots$ & -8.6 & -7.6 & 6.6 & 36.8 & 0.10 & 0.355 & 0.444 \\
\hline & & \multicolumn{2}{|c|}{$6.7-8.8$} & $\ldots$ & $\ldots$ & $-9.6^{g}$ & -7.8 & 4.3 & $22.6^{g}$ & 0.00 & 0.427 & 0.427 \\
\hline & & $6.7-8.4$ & $8.4-9.3$ & 7 & 1.0 & -8.9 & -7.6 & 5.5 & 29.3 & 0.00 & 0.418 & 0.418 \\
\hline & & $6.7-8.4$ & $9.4-10$ & 20 & 0.6 & -8.4 & -7.5 & 6.3 & 33.0 & 0.00 & 0.412 & 0.412 \\
\hline & & $6.75-8.4$ & $9.4-10$ & 1000 & 32.5 & -8.7 & -7.6 & 5.4 & 30.5 & 0.12 & 0.361 & 0.468 \\
\hline & \multirow[t]{4}{*}{ a4 } & \multicolumn{2}{|c|}{$7.1-8.2$} & $\ldots$ & $\ldots$ & -8.9 & -7.9 & 0.6 & 3.4 & 0.00 & 0.350 & 0.350 \\
\hline & & $7.1-8.2$ & $8.2-9.3$ & 100 & 8.1 & -8.9 & -7.9 & 0.7 & 3.8 & 0.00 & 0.358 & 0.358 \\
\hline & & $7.1-7.6$ & 8.9-9.3 & 19 & 0.5 & $-6.8^{g}$ & -5.6 & 1.7 & $9.7^{g}$ & 0.00 & 0.342 & 0.342 \\
\hline & & $7.1-8.2$ & $9.4-10$ & 1000 & 19.7 & -8.8 & -7.9 & 0.7 & 3.9 & 0.00 & 0.352 & 0.352 \\
\hline \multicolumn{13}{|c|}{$\begin{array}{l}{ }^{a} \text { Duration of } \mathrm{SF} \text { in } \log t(t \text { in } \mathrm{yr}) . \\
{ }^{b} b \equiv \mathrm{SFR} \text { (young)/SFR(old). } \\
{ }^{c} E W \text { in } \AA \text {. } \\
{ }^{\mathrm{d}} \text { Intrinsic colour is that for the stellar population in the absence of extinction. }(V-I)_{\text {reddened }}=(V-I)_{\text {intrinsic }}+0.891 C(\mathrm{H} \beta) . \\
{ }^{e} \text { Slit orientation with position angle } \mathrm{PA}=22^{\circ} . \\
{ }^{f} \text { Slit orientation with position angle } \mathrm{PA}=48^{\circ} .\end{array}$} \\
\hline
\end{tabular}

Since the contribution of ionized gas emission to the total brightness of region e1 is significant, the theoretical SED for this region has been constructed using a 4 Myr old stellar population SED for a heavy element mass fraction $Z=Z_{\odot} / 20$ to which the gaseous continuum SED and the observed emission lines were added (see Guseva et al. 2001). For comparison with the observed colour at PA $=22^{\circ}$, we reddened the predicted colour of region e1 by an amount corresponding to $C(\mathrm{H} \beta)=0.11$ (Table 3 in Thuan et al. 1999), and at PA $=48^{\circ}$ we adopted $C(\mathrm{H} \beta)=0$ (Table 1). The observed colour of region e1 is very blue, $(V-I) \sim-0.4$ (Figs. $8 \mathrm{~b}$ and d), and cannot be reproduced by a $4 \mathrm{Myr}$ old stellar population alone ( $(V-$ $I) \sim 0.1)$. Strong gaseous continuum and line emission need to be added (Table 1).

On the other hand, in all the LSB regions the contribution of the gaseous emission to the total flux is negligible. In Figs. 8b and $d$ we show by different symbols the colours of the LSB regions with various star formation history (Table 5). The total colour (stellar plus gaseous emission) of region e1 is shown by a filled squares. The agreement between the $(V-I)$ colours and those derived from the spectral energy distributions is very good. However, some uncertainties in the colours may be introduced by the uncertainties in the reddening curves and the ratios of total to selective extinction $R=A(V) / E(B-V)$. We use $R=3.2$ by Aller (1984), based on the reddening curve by Seaton (1979). Schlegel et al. (1998) give a slightly different $R=3.315$. Nevertheless, because the extinction in the studied regions is small, the errors introduced by the use of different reddening curves and $R$ are less than $2 \%$ in the $V$ band and negligible in the $I$ band. The $(B-V)$ colour of $\sim 0.2 \mathrm{mag}$, derived from the SEDs of the LSB regions, is also consistent with the observed value, derived from the $B$ and $V$ SBPs, which are shown in Fig. 3a.

\subsection{Comparison of the LSB component photometric properties for galaxies with different metallicities}

Our comprehensive studies of selected galaxies with oxygen abundances $12+\log (\mathrm{O} / \mathrm{H}) \lesssim 7.6$ and blue LSB components (SBS 0335-052, Izotov et al. 1997b, Papaderos et al. 1998; I Zw 18, Izotov et al. 2001b, Papaderos et al. 2002; SBS 0940+544, Guseva et al. 2001; Tol 1214-277, 
Table 6. $(V-I)$ colours of the extended LSB components in some irregular and BCD galaxies.

\begin{tabular}{llccllrr}
\hline \hline No. & Galaxy & $(V-I)_{\text {LSB }^{a}}$ & Telescope & $12+\log (\mathrm{O} / \mathrm{H})$ & Telescope $^{c}$ & Ref. $^{d}$ & Ref. $^{e}$ \\
\hline 1 & NGC 6789 & 0.98 & $H S T$ & 7.7 or 8.5 & - & 19 & 2 \\
2 & He 2-10 & 0.95 & $H S T$ & $7.9^{* * *}$ & $4 \mathrm{~m}$ & 19 & 11 \\
3 & NGC 625 & 0.90 & $H S T$ & $\sim 8.2^{*}$ & $1.6 \mathrm{~m}$ & 19 & 16 \\
4 & Mrk 996 & 0.90 & $H S T$ & 8.1 & $H S T$ & 15 & 15 \\
5 & VII Zw 403 & 0.90 & $H S T$ & 7.73 & $4 \mathrm{~m}$ & 13 & 9 \\
6 & Mrk 209 & 0.88 & $2.1 \mathrm{~m}$ & 7.77 & $4 \mathrm{~m}$ & 19 & 8 \\
7 & UGCA 290 & 0.88 & $H S T$ & $\sim 7.6$ & $H S T$ & 19 & 1 \\
8 & NGC 4214 & 0.86 & $H S T$ & 8.2 & $2.1 \mathrm{~m}$ & 19 & 10 \\
9 & SBS 1437+370 & 0.83 & $2.1 \mathrm{~m}$ & 7.93 & $4 \mathrm{~m}$ & 19 & 8 \\
10 & SBS 1319+579 & 0.83 & $2.1 \mathrm{~m}$ & 8.11 & $4 \mathrm{~m}$ & 19 & 8 \\
11 & NGC 1705 & 0.83 & $H S T$ & 8.36 & $1 \mathrm{~m}$ & 19 & 14 \\
12 & NGC 2366 & 0.80 & $H S T$ & 7.9 & $4 \mathrm{~m}$ & 19 & 8 \\
13 & UGC 4483 & 0.70 & $H S T$ & 7.58 & $4 \mathrm{~m}$ & 7 & 8 \\
14 & SBS 1249+493 & 0.68 & $2.1 \mathrm{~m}$ & 7.72 & $4 \mathrm{~m}$ & 19 & 8 \\
15 & SBS 1331+493 & 0.67 & $2.1 \mathrm{~m}$ & 7.77 & $4 \mathrm{~m}$ & 19 & 8 \\
16 & SBS 0940+544 & 0.58 & $2.1 \mathrm{~m}$ & 7.50 & Keck & 3 & 3 \\
17 & SBS 1129+576 & 0.56 & $2.1 \mathrm{~m}$ & 7.42 & $4 \mathrm{~m}$ & 17 & 17 \\
18 & Tol 65 & 0.50 & $H S T$ & 7.54 & Keck & 19 & 4 \\
19 & CG 1116+51 & 0.50 & $2.1 \mathrm{~m}$ & 7.5 & MMT & 19 & 19 \\
20 & HS 1442+4250 & 0.40 & $2.1 \mathrm{~m}$ & 7.63 & $4 \mathrm{~m}$ & 18 & 18 \\
21 & SBS 1415+437 & 0.40 & $2.1 \mathrm{~m}$ & 7.61 & $4 \mathrm{~m}$ & 19 & 19 \\
22 & Tol 1214-277 & 0.40 & $H S T$ & 7.54 & Keck & 19 & 4 \\
23 & Pox 186 & 0.40 & $H S T$ & 7.73 & MMT & 19 & 19 \\
24 & I Zw 18C & 0.10 & $H S T$ & $7.2^{* *}$ & Keck & 5 & 5 \\
25 & SBS 0335-052 & 0.0 & $H S T$ & 7.30 & Keck & 12 & 6 \\
26 & IZw 18 & 0.0 & $2.1 \mathrm{~m}, H S T$ & 7.2 & Keck & 13 & 6 \\
\hline & & & & & & 19 \\
\end{tabular}

${ }^{a}$ Colour corrected for reddening in our Galaxy. $E(V-I)_{\text {Galaxy }}$ are taken from the NED.

${ }^{b}$ Telescope used to obtain photometry.

${ }^{c}$ Telescope used to obtain spectroscopic data.

${ }^{d}$ Reference for the photometry.

${ }^{e}$ Reference for the chemical abundance.

* Our rough estimate.

** Oxygen abundance of the main component I Zw $18 \mathrm{NW}+\mathrm{SE}$.

*** Oxygen abundance is calculated using the observed data by Kobulnicky et al. (1999) and calibration by Pilyugin (2000).

References: (1) Crone et al. (2002); (2) Drozdovsky et al. (2001); (3) Guseva et al. (2001); (4) Izotov et al. (2001a); (5) Izotov et al. (2001b); (6) Izotov et al. (1999); (7) Izotov \& Thuan (2002); (8) Izotov \& Thuan (1999); (9) Izotov et al. (1997a); (10) Kobulnicky \& Skillman (1996); (11) Kobulnicky et al. (1999); (12) Papaderos et al. (1998); (13) Papaderos et al. (2002); (14) Storchi-Bergmann et al. (1994); (15) Thuan et al. (1996); (16) Vaceli et al. (1997); (17) Guseva et al. (2003a); (18) Guseva et al. (2003b); (19) this paper.

Fricke et al. 2001; Tol 65, Papaderos et al. 1999; SBS 1129+576, Guseva et al. 2003a; HS 1442+4250, Guseva et al. 2003b; SBS $1415+437$, this paper) have led us to the conclusion that these galaxies might be young. This is in contrast to the large age estimates for some well-studied higher-metallicity irregular and BCD galaxies, such as VII Zw 403 and UGCA 290 (Schulte-Ladbeck et al. 1998; Crone et al. 2002). To investigate this apparent inconsistency we therefore compare the $(V-I)$ colours of the LSB components $\left((V-I)_{\mathrm{LSB}}\right)$ of the galaxies from our sample with those in galaxies where a large age was derived from colour-magnitude diagrams (CMD).

In Table 6, we show the oxygen abundances and $(V-$ I) colours of the LSB components in several dwarf irregular and BCD galaxies. The telescopes used for photometric and spectroscopic observations are also given in the table. Ground-based photometric data in $V$ and $I$ were obtained with the $2.1 \mathrm{~m}$ KPNO telescope by Y. Izotov and R. Green. The HST photometric data were retrieved from the archive of the 


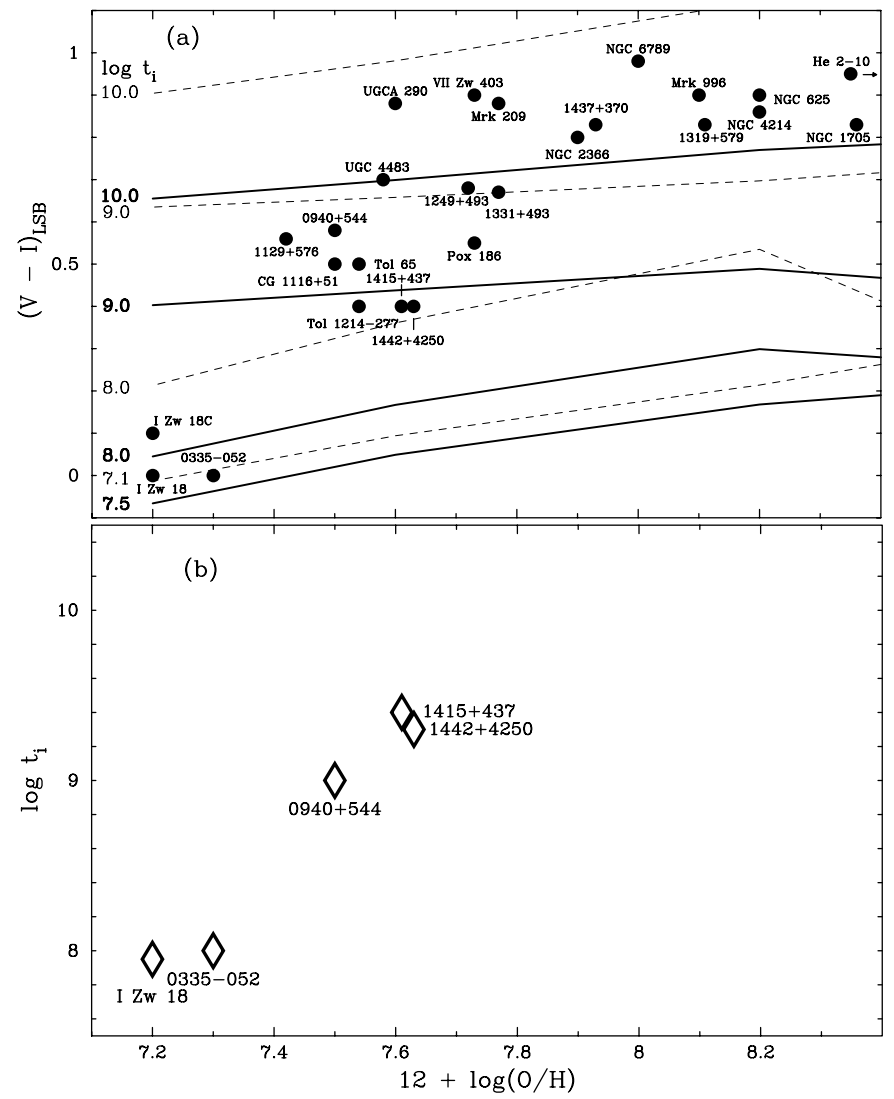

Fig. 9. Dependence of the $(V-I)$ colours of the LSB component a) and logarithm of the derived ages $\mathbf{b}$ ) on the oxygen abundance for the dwarf irregular and BCD galaxies from our sample. Dashed lines in a) show theoretical dependences of the $(V-I)$ colour on the oxygen abundance for an instantaneous burst calculated using the galactic evolution code PEGASE.2 (Fioc \& Rocca-Volmerange 1997) in the range of ages (in logarithmic scale) $\log t_{\mathrm{i}}$ between 7.1 and $10.0\left(t_{\mathrm{i}}\right.$ in yr). Solid lines show models in which stars are forming continuously at a constant SFR between $t_{\mathrm{f}}=0$ and different $t_{\mathrm{i}}$, where $\log t_{\mathrm{i}}$ varies between 7.5 and 10.0. The observed colours are corrected only for reddening in our Galaxy. The ages in b) are from Izotov et al. (2001b) (I Zw 18), Papaderos et al. (1998) (SBS 0335-052), Guseva et al. (2001) (SBS 0940+544), Guseva et al. (2003b) (HS 1442+4250) and this paper (SBS 1415+437).

Space Telescope Science Institute $(\mathrm{STScI})^{5}$. We included in the sample only galaxies with low internal and/or foreground interstellar extinction to avoid uncertainties introduced by the correction of the $(V-I)$ colour for reddening. Thus, we do not include the nearby star-forming galaxy NCG 1569 studied with $H S T$, for example. The chemical abundances for the majority of galaxies are obtained from spectroscopic observations of their $\mathrm{H}$ II regions. An exception is I Zw 18C, the faint component of the BCD I Zw 18, where no emission lines of heavy elements were detected. Therefore, for I Zw $18 \mathrm{C}$ we adopt the oxygen abundance derived for the bright main body of I Zw 18. $(V-I)$ colours of the galaxies in Table 6 are corrected for reddening

${ }^{5}$ STScI is operated by the AURA, Inc., under NASA contract NAS5-26555. in our Galaxy, with $E(V-I)_{\text {Galaxy }}$ taken from the NASA/IPAC Extragalactic Database (NED).

The dependence of the $(V-I)_{\text {LSB }}$ colours on oxygen abundance for 26 selected galaxies is shown in Fig. 9a. Thin dashed lines represent theoretical dependences for an instantaneous burst in the age range from $\log t_{\mathrm{i}}=7.1$ to $\log t_{\mathrm{i}}=10.0\left(t_{\mathrm{i}}\right.$ in yr). The models for continuous star formation are shown by thick solid lines. These models are calculated for a constant SFR which started at time $t_{\mathrm{i}}$, with $\log t_{\mathrm{i}}$ between 7.5 and 10.0 and continuing until now $\left(t_{\mathrm{f}}=0\right)$. The blueing of the $(V-I)$ colour with decreasing oxygen abundance is in agreement with model predictions. However, this blueing trend for galaxies with $12+\log (\mathrm{O} / \mathrm{H}) \lesssim 7.6$ is too steep to be explained only by metallicity effects. The blue colours of the LSB components of these low-metallicity BCDs are also not due to ionized gas emission, as the latter dominates the outer parts of only two BCDs: I Zw 18 (Izotov et al. 2001b; Papaderos et al. 2002) and SBS 0335-052 (Izotov et al. 1997b; Papaderos et al. 1998). In other galaxies, including I Zw 18C, the LSB component emission has mainly a stellar origin. The steep trend cannot be explained by reddening effects because the interstellar extinction derived from the spectroscopic observations is small for the galaxies shown in Fig. 9a. Therefore, it is likely that the blueing is mainly due to a change in the age of the stellar populations, and that low-metallicity galaxies are younger than high-metallicity ones. All objects with $12+\log (\mathrm{O} / \mathrm{H}) \lesssim 7.6$ and $(V-I)_{\text {LSB }} \lesssim 0.6 \mathrm{mag}$ are in the range of colours predicted for ages $\lesssim 1-2$ Gyr by instantaneous and continuous models of star formation. On the other hand, galaxies with $\left(V_{-}\right.$ $I)_{\text {LSB }} \gtrsim 0.7$ mag are likely to be older, with ages $\sim 10 \mathrm{Gyr}$. Yi (2003) has analysed the uncertainties in the synthetic integrated $(V-I)$ colours caused by uncertainties in the stellar evolutionary models, population synthesis techniques and stellar atmosphere models. He has shown that, despite all the uncertainties, a $(V-I) \lesssim 0.65-0.75$ mag can be attributed to an intermediateage stellar population with an age not larger than $2 \mathrm{Gyr}$, in the case of an instantaneous burst of star formation, in agreement with the age derived here from the PEGASE. 2 models (dashed lines in Fig. 9a). The smaller luminosity-weighted age of galaxies with blue LSB components in Fig. 9a is supported by the detailed analysis of the spectroscopic and photometric properties of some of these galaxies, as demonstrated in this series of papers.

Izotov \& Thuan (1999) have suggested that the oxygen abundance may be a good age indicator. That this appears to be the case is shown in Fig. 9b where a clear trend of increasing age of the LSB stellar population with increasing oxygen abundance is seen for 5 star-forming galaxies with available data. The ages for 4 galaxies (I Zw 18, Izotov et al. 2001b; SBS 0940+544, Guseva et al. 2001; HS 1442+4250, Guseva et al. 2003b; SBS 1415+437, this paper) are determined by the four methods described before. The age for SBS 0335052 (Papaderos et al. 1998) is derived using only colours and SEDs. This is because its LSB component is embedded into the $\mathrm{H}$ II region produced by the young central clusters and no absorption lines are present in the spectra. 


\section{Conclusions}

The results of a detailed photometric and spectroscopic study of the metal-deficient blue compact dwarf galaxy SBS $1415+437$ are presented. $\mathrm{H} \alpha$ images and spectra in the optical range have been obtained with the Kitt Peak $2.1 \mathrm{~m}$ and $4 \mathrm{~m}$ telescopes, respectively. A $B$ image has been obtained with the $2.2 \mathrm{~m}$ Calar Alto telescope. These data are supplemented by $H S T /$ WFPC $2 V$ and $I$ images and MMT spectra from Thuan et al. (1999). The main conclusions of this study can be summarized as follows:

1. SBS $1415+437$ is a nearby $(D=11.4 \mathrm{Mpc})$ low-metallicity cometary BCD with two bright $\mathrm{H}$ II regions in the SW part of the elongated low-surface-brightness (LSB) stellar component. The scale lengths $\alpha$ of the LSB component, obtained from surface brightness profiles (SBPs) in $V$ and $I$ are both $\sim 0.3 \mathrm{kpc}$, in excellent agreement with those in Thuan et al. (1999). The observed $(V-I)$ colour of the brightest $\mathrm{H}$ II region is very blue, $\sim-0.4 \mathrm{mag}$, due to the combined effects of a young stellar population and ionized gas emission. The colours of the low-surface-brightness component are much redder ( $\sim 0.4-0.5 \mathrm{mag})$ and roughly constant in the outer parts of the galaxy. A deeper $B$ band SBP of SBS $1415+437$ reveals at intermediate and large radii two exponential intensity regimes with a scale length of 0.3 and $0.27 \mathrm{kpc}$, respectively. The inner one $\left(4^{\prime \prime} \lesssim R^{*} \lesssim\right.$ $\left.13^{\prime \prime}\right)$ can be identified with the exponential component derived from $H S T V$ and $I$ data. The outer one, which dominates for $R^{*} \gtrsim 16^{\prime \prime}$, is $\gtrsim 0.3$ mag fainter than the inner component.

2. In the two brightest $\mathrm{H}$ II regions we derive oxygen abundances of $12+\log (\mathrm{O} / \mathrm{H})=7.61 \pm 0.01$ and $7.62 \pm 0.03$ $\left(Z \sim Z_{\odot} / 20\right)$. These values agree well with previous determinations (Thuan et al. 1999). Other heavy element-tooxygen abundance ratios for these $\mathrm{H}$ II regions are also in good agreement with mean ratios derived from previous studies of BCDs (Thuan et al. 1995; Izotov \& Thuan 1999).

3. The ${ }^{4} \mathrm{He}$ mass fractions $Y=0.246 \pm 0.003$ and $0.243 \pm 0.010$, derived for the brightest H II regions of SBS $1415+437$, are in good agreement with previous determinations for this galaxy and with the primordial ${ }^{4} \mathrm{He}$ mass fraction $Y_{\mathrm{p}}=(0.244-0.245) \pm 0.002$ (Izotov \& Thuan 1998; Izotov et al. 1999).

4. We use four different methods and different SF scenarios to derive the age of the stellar populations in the LSB component of the galaxy. For the outer LSB regions, the equivalent widths of the $\mathrm{H} \alpha$ and $\mathrm{H} \beta$ emission lines, the equivalent widths of the $\mathrm{H} \gamma$ and $\mathrm{H} \delta$ absorption lines, the spectral energy distributions and the $(V-I)$ colours are reproduced quite well by models in which only a young stellar population $(t \lesssim 250 \mathrm{Myr})$ is present. For those regions, only a small extinction with $C(\mathrm{H} \beta)$ in the range $0-0.1$ is needed. An older stellar population, if present, does not contribute substantially to the optical luminosity of those outer regions. For region a1, located closer to the brightest $\mathrm{H}$ II region, a larger extinction $(C(\mathrm{H} \beta)=0.22)$ is required to fit the observational data by the same young stellar population. The assumption of $C(\mathrm{H} \beta)=0$ in all regions would require an increase of the relative fraction of the old stellar population from the outer LSB parts to the inner bright $\mathrm{H}$ II regions, which would be contrary to the trend observed in the majority of BCDs, where the relative contribution of old stars increases outwards.

Assuming no extinction, we find that the upper limit to the mass of the old stellar population in SBS 1415+437, formed between $2.5 \mathrm{Gyr}$ and $10 \mathrm{Gyr}$, is not greater than $\sim(1 / 20-1)$ of the mass of the stellar population formed during the last $\sim 250 \mathrm{Myr}$. Depending on the region considered, this also implies that the star formation rate during the most recent star formation in SBS $1415+437$ must be 20 to 1000 times greater than the SFR at ages $\gtrsim 2.5 \mathrm{Gyr}$.

5. We compare the $(V-I)$ colours of the LSB components and the oxygen abundances of a sample of 26 lowmetallicity dwarf irregular and BCD galaxies, including SBS $1415+437$. It is shown that the LSB components are systematically bluer in the lower-metallicity galaxies. However, the observed trend is too steep to be explained only by metallicity effects. Therefore, it is likely that lowermetallicity galaxies have also younger populations. In particular, the luminosity-weighted ages of galaxies with LSB colours of $(V-I) \lesssim 0.6$ are probably not greater than 1-2 Gyr.

Acknowledgements. N.G.G. has been supported by DFG grant 436 UKR 17/2/02 and Y.I.I. acknowledges the Göttingen Academy of Sciences for a Gauss professorship. N.G.G. and Y.I.I. have been partially supported by Swiss SCOPE 7UKPJ62178 grant. They are grateful for the hospitality of the Göttingen Observatory. Y.I.I. and T.X.T. have been partially supported by NSF grant AST-02-05785. Research by P.P. and K.J.F. has been supported by the Deutsches Zentrum für Luft- und Raumfahrt e.V. (DLR) under grant 50 OR 9907 7. K.G.N. acknowledges the support from the Deutsche Forschungsgemeinschaft (DFG) grants FR 325/50-1 and FR 325/50-2. This research has made use of the NASA/IPAC Extragalactic Database (NED) which is operated by the Jet Propulsion Laboratory, California Institute of Technology, under contract with the National Aeronautics and Space Administration.

\section{References}

Aller, L. H. 1984, Physics of Thermal Gaseous Nebulae (Dordrecht: Reidel)

Anders, E., \& Grevesse, N. 1989, Geochim. Cosmochim. Acta, 53, 197

Bessell, M. S. 1990, PASP, 102, 1181

Bessell, M. S., Castelli, F., \& Plez, B. 1998, A\&A, 333, 231

Bica, E., \& Alloin, D. 1986, A\&AS, 66, 171

Christian, C. A., Adams, M., Barnes, J. V., et al. 1985, PASP, 97, 363

Crone, M. M., Schulte-Ladbeck, R. E., Greggio, L., \& Hopp, U. 2002, ApJ, 567, 258

Drozdovsky, I. O., Schulte-Ladbeck, R. E., Hopp, U., Crone, M. M., \& Greggio, L. 2001, ApJ, 551, L135

Esteban, C., Peimbert, M., Torres-Peimbert, S., \& Escalante, V. 1998, MNRAS, 295, 401

Esteban, C., Peimbert, M., Torres-Peimbert, S., Garcia-Rojas, J., \& Rodriguez, M. 1999a, ApJS, 120, 113

Esteban, C., Peimbert, M., Torres-Peimbert, S., \& Garcia-Rojas, J. 1999b, Rev. Mex. Astron. Astrofiz., 35, 65

Fioc, M., \& Rocca-Volmerange, B. 1997, A\&A, 326, 950 
Fricke, K. J., Izotov, Y. I., Papaderos, P., Guseva, N. G., \& Thuan, T. X. 2001, AJ, 121, 169

González Delgado, R. M., Leitherer, C., \& Heckman, T. M. 1999, ApJS, 125, 489

Guseva, N. G., Izotov, Y. I., Papaderos, P., et al. 2001, A\&A, 378, 756 Guseva, N. G., Papaderos, P., Izotov, Y. I., et al. 2003a, A\&A, 407, 75 Guseva, N. G., Papaderos, P., Izotov, Y. I., et al. 2003b, A\&A, 407, 91 Izotov, Y. I., \& Thuan, T. X. 1998, ApJ, 500, 188

Izotov, Y. I., \& Thuan, T. X. 1999, ApJ, 511, 639

Izotov, Y. I., \& Thuan, T. X. 2002, ApJ, 567, 875

Izotov, Y. I., Thuan, T. X., \& Lipovetsky, V. A. 1994, ApJ, 435, 647 Izotov, Y. I., Thuan, T. X., \& Lipovetsky, V. A. 1997a, ApJS, 108, 1 Izotov, Y. I., Lipovetsky, V. A., Chaffee, F. H., et al. 1997b, ApJ, 476, 698

Izotov, Y. I., Chaffee, F. H., Foltz, C. B., et al. 1999, ApJ, 527, 757

Izotov, Y. I., Chaffee, F. H., \& Green, R. F. 2001a, ApJ, 562, 727

Izotov, Y. I., Chaffee, F. H., Foltz, C. B., et al. 2001b, ApJ, 560, 222

Kobulnicky, H. A., \& Skillman, E. D. 1996, ApJ, 471, 211

Kobulnicky, H. A., Kennicutt, R. C., Jr., \& Pizagno, J. L. 1999, ApJ, 514,544

Noeske, K. G., Guseva, N. G., Fricke, K. J., et al. 2000, A\&A, 361, 33
Papaderos, P., Loose, H.-H., Thuan, T. X., \& Fricke, K. J. 1996, A\&AS, 120, 207

Papaderos, P., Izotov, Y. I., Fricke, K. J., Thuan, T. X., \& Guseva, N. G. 1998, A\&A, 338, 43

Papaderos, P., Fricke, K. J., Thuan, T. X., Izotov, Y. I., \& Nicklas, H. 1999, A\&A, 352, 57

Papaderos, P., Izotov, Y. I., Thuan, T. X., et al. 2002, A\&A, 393, 461

Pilyugin, L. S. 2001, A\&A, 369, 594

Seaton, M. J. 1979, MNRAS, 187, 73

Schlegel, D. J., Finkbeiner, D. P., \& Davis, M. 1998, ApJ, 500, 525

Schulte-Ladbeck, R. E., Crone, M. M., \& Hopp, U. 1998, ApJ, 493, 23

Storchi-Bergmann, T., Calzetti, D., \& Kinney, A. L. 1994, ApJ, 429, 572

Thuan, T. X., Izotov, Y. I., \& Lipovetsky, V. A. 1995, ApJ, 445, 108

Thuan, T. X., Izotov, Y. I., \& Lipovetsky, V. A. 1996, ApJ, 463, 120

Thuan, T. X., Izotov, Y. I., \& Foltz, C. B. 1999, ApJ, 525, 105

Vaceli, M. S., Viegas, S. M., Gruenwald, R., \& De Souza, R. E. 1997, AJ, 114, 1345

Yi, S. K. 2003, ApJ, 582, 202 